
\title{
Dual-Layered MIMO Transmission for Increased Bandwidth Efficiency
}

\author{
Christos Masouros, Senior Member, IEEE, and Lajos Hanzo, Fellow, IEEE
}

\begin{abstract}
4 Abstract-A dual-layered downlink transmission scheme is 5 proposed for intrinsically amalgamating multiple-input-multiple6 output (MIMO) spatial multiplexing (SMX) with spatial modu7 lation (SM). The proposed scheme employs a classic SMX trans8 mission that is known to offer superior bandwidth efficiency (BE) 9 compared with SM. We exploit receive-antenna-based SM (RSM) 10 on top of this transmission as an enhancement of the BE. The RSM 11 here is applied to the combined spatial and power-level domain not 12 by activating and deactivating the RAs but rather by choosing be13 tween two power levels $\left\{\boldsymbol{P}_{\boldsymbol{1}}, \boldsymbol{P}_{\boldsymbol{2}}\right\}$ for the received symbols in these 14 antennas. In other words, the combination of symbols received at 15 a power level $\boldsymbol{P}_{\boldsymbol{1}}$ carries information in the spatial domain in the 16 same manner as the combination of nonzero elements in the re17 ceive symbol vector carries information in the RSM transmission. 18 This allows for the coexistence of RSM with SMX, and the results 19 show increased BE for the proposed scheme compared with both 20 SMX and SM. To characterize the proposed scheme, we carry out 21 a mathematical analysis of its performance, and we use this to 22 optimize the ratio between $\boldsymbol{P}_{1}$ and $\boldsymbol{P}_{2}$ for attaining the minimum 23 error rates. Our analytical and simulation results demonstrate 24 significant BE gains for the proposed scheme compared with 25 conventional SMX and SM.
\end{abstract}

26 Index Terms-Multiple-input-multiple-output systems, spatial 27 modulation (SM), spatial multiplexing (SMX), transmit precoding 28 (TPC).

\section{INTRODUCTION}

${ }^{n} \mathbf{M}$ ULTIANTENNA-aided transceivers have been shown to improve the capacity of the wireless channel by 33 means of spatial multiplexing (SMX) [1]. For the multiuser 34 downlink (DL), transmit precoding (TPC) schemes have been 35 shown to improve both the attainable power efficiency (PE) and 36 the cost of mobile terminals by shifting the signal processing 37 complexity to the base stations (BSs). Numerous TPC solutions

Manuscript received November 25, 2014; revised February 16, 2015 and April 20, 2015; accepted May 22, 2015. The work of C. Masouros was supported in part by the Royal Academy of Engineering, U.K., and in part by the Engineering and Physical Sciences Research Council (EPSRC) under Project EP/M014150/1. The work of L. Hanzo was supported in part by the India-U.K. Advanced Technology Centre, by the EU Concerto Project, by the European Research Council under the Advanced Fellow Grant, and by the Royal Society under the Wolfson Research Merit Award. The review of this paper was coordinated by Dr. N.-D. Đào.

C. Masouros is with the Department of Electrical and Electronic Engineering, University College London, London WC1E 7JE, U.K. (e-mail: chris. masouros@ieee.org).

L. Hanzo is with the School of Electronics and Computer Science, University of Southampton, Southampton SO17 1BJ, U.K. (e-mail: 1h@ecs.soton.ac.uk).

Color versions of one or more of the figures in this paper are available online at http://ieeexplore.iee.org.

Digital Object Identifier 10.1109/TVT.2015.2438776 exist, ranging from highly complex capacity achieving nonlin- 38 ear dirty paper coding techniques [2] and their low-complexity 39 suboptimal counterparts in the form of Tomlinson-Harashima 40 precoding [3]-[6] to linear TPC schemes based on channel 41 inversion [7]-[12] that offers the lowest complexity, albeit at an 42 inferior performance. The performance-complexity tradeoffs 43 between the above TPC have been thoroughly studied in the 44 literature. More recently, it has been shown that the family 45 of linear techniques exhibits a close-to-optimal performance 46 in the large-scale multiple-input-multiple-output (MIMO) 47 region [13]-[15]. Accordingly, we focus on the class of low- 48 complexity closed-form linear TPC [7]-[12] due to their favor- 49 able performance-complexity tradeoff and practical relevance. 50

More recently, spatial modulation (SM) has been conceived 51 for implicitly encoding information in the index of the spe- 52 cific antenna activated for the transmission of the modulated 53 symbols, offering a low-complexity design alternative [16]. Its 54 central benefits include the absence of interantenna interference 55 (IAI) and the fact that it only requires a subset (down to 56 one) of radio-frequency (RF) chains compared with SMX. 57 Accordingly, the interantenna synchronization is also relaxed. 58 Early work has focused on the design of receiver algorithms for 59 minimizing the bit error ratio (BER) of SM at low complexity 60 [16]-[21]. The work spans from matched filtering as a low- 61 complexity technique for detecting the antenna index used for 62 SM [16] to the maximum likelihood (ML) [20] with a signif- 63 icantly reduced complexity compared with classic SMX ML 64 detectors, including compressive sensing approaches [18] and 65 performance analyses [19]. Reduced-space sphere detection has 66 also been proposed for SM in [21] for further complexity reduc- 67 tion where a generalized SM transmission was also explored 68 [22]. In addition to receive processing, recent work has also 69 proposed constellation shaping for SM [23]-[33]. Specifically, 70 the work on this topic has focused on three main directions: 71 shaping and optimization of the spatial constellation, i.e., the le- 72 gitimate sets of activated transmit antennas (TAs) [23], modula- 73 tion constellation shaping [24]-[28] for the SM and space shift 74 keying transmission, where the constellation of the modulated 75 bits is optimized, and joint spatial and modulation constellation 76 shaping, in the form of optimizing the received constellation 77 [29]-[33].

Closely related work has focused on applying the concept of 79 $\mathrm{SM}$ to the receive antennas (RAs) of the communication link, 80 as opposed to the TAs as per the above approaches, forming 81 the RA-based spatial modulation (RSM) concept [36]-[39]. By 82 means of TPC, this technique targets a specific subset of RAs, 83 which receive information symbols, whereas the rest of the RAs 84 
85 receive only noise. This may be achieved by using zero-forcing 86 (ZF) TPC and transmitting a combination of information sym87 bols and zeros to the RAs, depending on the spatial symbols 88 to convey. As opposed to conventional SM where a subset of $89 \mathrm{RF}$ chains is deployed, here, all TAs and RAs are active and 90 therefore there are no RF chain reductions. Still, the computa91 tional complexity of the receivers is drastically reduced, where 92 simply the indexes of the targeted RAs have to be detected, 93 and the classic symbols received at the activated RAs are then 94 demodulated.

95 Inspired by the above RSM philosophy, here, we propose 96 a dual-layered transmission (DLT) scheme, which intrinsically 97 amalgamates a full spatial multiplexing (SMX) with SM. First, 98 we note that since, for RSM, all TAs and RAs are active, there 99 are no RF chain reductions, and this motivates the full SMX 100 approach. To accommodate the SMX, we apply an SM to the 101 combined spatial and receive-power domain, where instead of 102 sending a combination of information symbols and zero power 103 to the RAs, we apply two different power levels for distinguish104 ing between the "active" and "inactive" RAs. In this manner, the 105 spatial symbols are formed based on the power levels detected. 106 We demonstrate that this improves the bandwidth efficiency 107 (BE) with respect to SMX and SM. Against this state of the 108 art, we list the main contributions of this paper.

110 - We propose a new DLT scheme based on linear TPC that 111 improves the BE by jointly exploiting the benefits of SMX 112 and RSM.

113

- We provide the performance analysis of the proposed technique based on the pairwise error probability (PEP) between different constellation points in the supersymbol constellation formed by the combination of the spatial constellation of RSM and the classic modulation constellation of SMX.

- We use the above results for analytically deriving the optimum power ratio between the two sets of antennas that carry the spatial symbol for the proposed scheme for minimizing the probability of detection errors.

- We calculate and compare analytically the complexity of the conventional and proposed techniques, and quantify the performance-complexity tradeoff of conventional and proposed schemes, by introducing a PE metric that combines the BE, transmit power, and complexity, to prove the enhanced tradeoff for the proposed scheme.

Remark 1: It should be noted that, while this paper focuses 130 on a single-link scenario, the proposed technique can be readily 131 extended to a multiuser DL scenario, where the DLT and the 132 related RSM take place on a per-user basis, as facilitated by the 133 ZF-TPC employed at the BS.

134 Remark 2: The proposed scheme does not consist of a power 135 allocation scheme in the sense of allocating power according 136 to the quality-of-service $(\mathrm{QoS})$ requirements of the user. This 137 power allocation may be applied in addition to the proposed 138 scheme in the multiuser scenario, where different users with 139 different QoS requirements employ different sets of power 140 levels $\left\{P_{1}, P_{2}\right\}$ accordingly.

141 Remark 3: To facilitate the proposed power-level modula142 tion, this paper focuses on phase shift keying (PSK) in terms of the classical symbol modulation. Its adaptation to quadrature 143 amplitude modulation (QAM) is not trivial since the variability 144 of the power levels for the classically modulated symbols 145 would hinder the detection of the power levels of the spatially 146 modulated symbols. Nevertheless, even for PSK modulation, 147 our results illustrate a wide range of achievable BEs for the 148 proposed scheme and an improved performance compared with 149 classical SMX associated with both PSK and QAM for the 150 same BE.

The remainder of this paper is organized as follows. Section II 152 presents the MIMO system model and introduces the RSM 153 transmission philosophy. Section III details the proposed DLT 154 scheme, whereas in Section IV, we present our analytical study 155 of the performance attained and the analytical optimization 156 of the power ratio for the proposed scheme. Section V detail 157 the complexity calculation and the study of the attainable PE. 158 Finally, Section VI presents our numerical results, whereas our 159 conclusions are offered in Section VII.

\section{System Model And Receive-Antenna-BAsed SPATIAL MOdUlation}

\section{A. System Model}

Consider a MIMO system, where the transmitter and receiver 164 are equipped with $N_{t}$ and $N_{r}$ antennas, respectively. For sim- 165 plicity, unless stated otherwise, in this paper, we assume that the 166 transmit power budget is limited as $P=1$. For the case of 167 the closed-form TPCs of [7]-[12], it is required that $N_{t} \geq N_{r} .168$ The given channel is modeled as follows:

$$
\mathbf{y}=\mathbf{H t}+\mathbf{w}
$$

where $\mathbf{y}$ is the vector of received symbols in all RAs, and $\mathbf{H}$ is 170 the MIMO channel vector with elements $h_{m, n}$ representing the 171 complex channel coefficient between the $n$th TA and the $m$ th 172 RA. Furthermore, $\mathbf{t}$ is the vector of precoded transmit symbols 173 that will be discussed in the following, and $\mathbf{w} \sim \mathcal{C N}\left(0, \sigma^{2} \mathbf{I}\right) 174$ is the additive white Gaussian noise (AWGN) component at 175 the receiver, with $\mathcal{C N}\left(\mu, \sigma^{2}\right)$ denoting the circularly symmet- 176 ric complex Gaussian distribution with a mean of $\mu$ and a 177 variance of $\sigma^{2}$.

\section{B. Receive-Antenna-Based Spatial Modulation}

The block diagram of RSM as proposed in [36] is shown 180 in Fig. 1(a). RSM targets a subset of the RAs by sending 181 information symbols to these RAs and zero power to the rest 182 of the RAs. While for RSM all RAs have to be on to detect 183 the arrival of information symbols, for coherence with the 184 SM literature, we shall refer to the antennas as "active" and 185 "inactive," depending on whether they do or do not receive 186 information symbols, respectively. The specific combination of 187 RAs that do receive symbols implicitly conveys the symbol 188 transmitted in the spatial domain. The above RA subset trans- 189 mission is achieved by forming a supersymbol vector in the 190 form $\mathbf{s}_{m}^{k}=\mathbf{e}_{k} b_{m}=\left[0, \ldots, b_{m_{1}}, \ldots, 0, \ldots, b_{m_{2}}, \ldots, 0\right]^{T}$ with 191 $N_{a}$ nonzero elements, where $\mathbf{e}_{k}$ is a diagonal matrix of size 192 


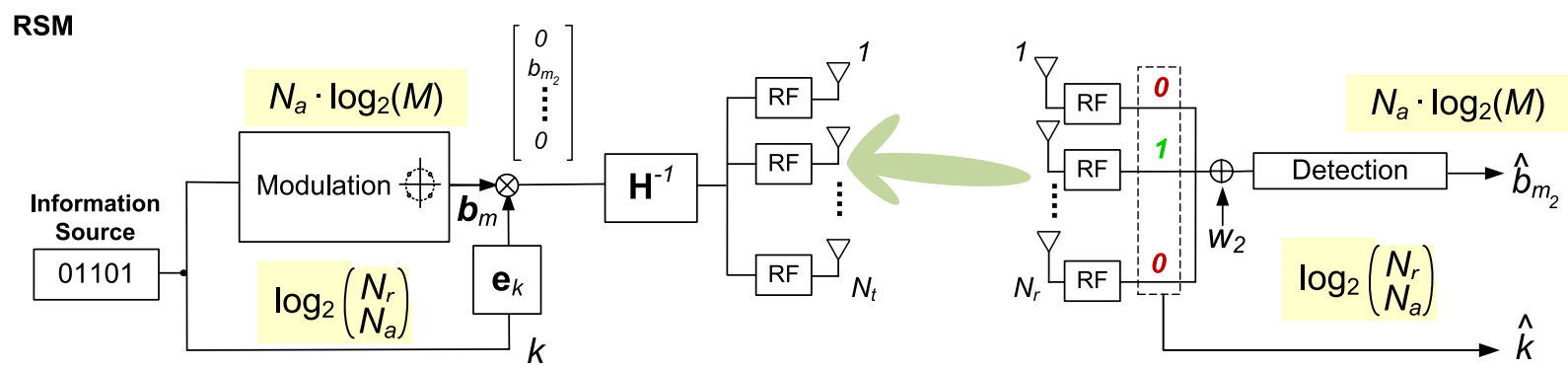

(a)

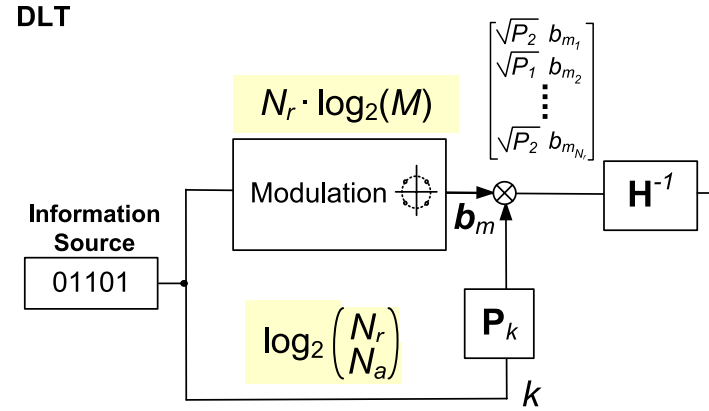

Fig. 1. Block diagram of (a) RSM and (b) DLT transmission.

$193 N_{r}$ with elements taken from the set $\{1,0\}$ on its diagonal, 194 which represents the RAs that are activated. The notation $[\cdot]^{T}$ 195 denotes the transpose operator. Here, $b_{m_{i}}, m_{i} \in\{1, \ldots, M\}$ 196 is a symbol taken from an $M$-order modulation alphabet that 197 represents the transmitted waveform in the baseband domain 198 conveying $\log _{2}(M)$ bits and $k$ represents the index of the $199 N_{a}$ activated RAs (the index of the nonzero elements in $\mathbf{s}_{m}^{k}$ ) 200 conveying $\log _{2}\left(\begin{array}{l}N_{r} \\ N_{a}\end{array}\right)$ bits in the spatial domain. Accordingly, 201 the total number of bits conveyed per supersymbol for RSM is

$$
\beta=N_{a} \log _{2}(M)+\log _{2}\left(\begin{array}{c}
N_{r} \\
N_{a}
\end{array}\right) .
$$

202 The transmitter then sends

$$
\mathbf{t}=f \mathbf{T} \mathbf{s}_{m}^{k}
$$

203 where $\mathbf{T}=\mathbf{H}^{H}\left(\mathbf{H H}^{H}\right)^{-1}$ is the ZF-TPC [7] that preserves 204 the form of $\mathbf{s}_{m}^{k}$ at the receiver. The factor $f=\sqrt{1 / \operatorname{tr}\left(\mathbf{T} \mathbf{T}^{H}\right)}$, 205 where $\operatorname{tr}(\cdot)$ denotes the trace operator and normalizes the 206 average transmit power to $P=1$. The received symbol vector 207 can be written as

$$
\mathbf{y}=f \mathbf{H T} \mathbf{s}_{m}^{k}+\mathbf{w}=f \mathbf{s}_{m}^{k}+\mathbf{w}
$$

208 where, clearly, all IAI is removed. At the receiver, a joint ML 209 detection of both the RA index and the transmit symbol is 210 obtained by the following minimization:

$$
\begin{aligned}
{\left[\hat{s}_{m}, \hat{k}\right] } & =\arg \min _{i}\left\|\mathbf{y}-\dot{\mathbf{y}}_{i}\right\| \\
& =\arg \min _{m_{i}, k_{i}}\left\|\mathbf{y}-f \mathbf{H T} \mathbf{s}_{m_{i}}^{k_{i}}\right\|
\end{aligned}
$$

211 where $\|\mathbf{x}\|$ denotes the norm of vector $\mathbf{x}$, and $\dot{\mathbf{y}}_{i}$ is the $i$ th 212 constellation point in the received SM constellation. A low- complexity decoupled approach is also proposed in [36], where 213 the first active antenna indexes are detected in the form of 214

$$
\hat{k}=\arg \max _{j \in \mathcal{J}} \sum_{i=1}^{N_{a}}\left|y_{j, i}\right|^{2}
$$

where $\mathcal{J}$ denotes the set of symbols in the spatial domain, and 215 then, the classic modulated symbols are detected by

$$
\hat{b}_{m_{i}}=\arg \min _{n_{i} \in \mathcal{Q}}\left|y_{\hat{k}, i} / f-b_{n_{i}}\right|^{2}
$$

where $\mathcal{Q}$ denotes the modulation constellation, and $b_{n_{i}}$ are 217 the symbols in the modulated symbol alphabet. For reasons of 218 computational complexity, we shall focus on the latter detector 219 in this paper.

\section{Proposed DuAL-LAYERED TRANSMISSION}

From the above system description, it can be seen that for 222 the particular case of RSM, while the detection complexity 223 is clearly reduced with respect to SMX, there are no savings 224 in RF complexity since all $N_{r}$ RAs have to be activated and 225 receiving for the detection in (6) and (7). Still, by forming a 226 subset of beams towards the receiver, as shown in Fig. 1(a), 227 the BE, i.e., the number of bits per channel use, is generally 228 lower for RSM than for SMX. Motivated by this, we propose 229 a dual-layered approach combining SMX with RSM, where 230 the BE of conventional SMX MIMO transmission is strictly 231 enhanced by encoding spatial bits in the RSM fashion in the 232 received power domain, by selecting two distinct, nonzero 233 power levels for the transmitted supersymbols instead of the 234 "on-off" RSM transmission in the $\{1,0\}$ manner. This allows 235 for nonzero elements throughout the supersymbol vector $\mathbf{s}_{m}^{k}, 236$ 
237 hence supporting a full SMX transmission in the modulated 238 signal domain. The block diagram of the proposed DLT is 239 shown in Fig. 1(b).

240 1) Transmitter: Here, we employ a full data vector in the 241 form of $\mathbf{b}_{m}=\left[b_{m_{1}}, b_{m_{2}}, \ldots, b_{m_{N_{r}}}\right]^{T}$, with all elements being 242 nonzero, and the encoding of the spatial bits is achieved by allo243 cating different power levels to the received symbols according 244 to the spatial symbol $k$, by applying the power allocation matrix $245 \mathbf{P}_{k}$, i.e.,

$$
\mathbf{s}_{m}^{k}=\mathbf{P}_{k} \mathbf{b}_{m}=\left[s_{m_{1}}, s_{m_{2}}, \ldots, s_{m_{N_{r}}}\right]^{T}
$$

246 with

$$
\mathbf{P}_{k}=\left[\begin{array}{cccc}
\sqrt{p_{1}} & 0 & \ldots & 0 \\
0 & \sqrt{p_{2}} & \cdots & 0 \\
\vdots & \vdots & \ddots & \vdots \\
0 & 0 & \ldots & \sqrt{p_{N_{r}}}
\end{array}\right]
$$

247 where $p_{i}, i \in\left[1, N_{r}\right]$ are taken from the set $\left\{P_{1}, P_{2}\right\}$ according 248 to the spatial symbol $k$. Note that classic QoS-based power al249 location can be applied in addition to this process by employing 250 an additional power allocation matrix on top of (9). The receiver 251 can then remove this additional matrix by simple inversion, in 252 order to detect the spatial symbol. For notational simplicity and 253 to keep the focus of the discussion on the proposed concept, we 254 neglect QoS-based power allocation.

255 2) Receiver: At the receiver side, the explicit knowledge of 256 the power levels $\left\{P_{1}, P_{2}\right\}$ is not required, as long as the detector 257 can distinguish between the two power levels. The received 258 signal of (4) can be decomposed as

$$
\begin{aligned}
& y_{p}=f \sqrt{P_{1}} b_{m_{p}}+w_{p}, p \in \mathcal{A} \\
& y_{q}=f \sqrt{P_{2}} b_{m_{q}}+w_{q}, q \in \mathcal{I}
\end{aligned}
$$

259 where $\mathcal{A}$ and $\mathcal{I}$ denote the sets of "active" and "inactive" 260 antennas, respectively. Hence, the receive processing is similar 261 to the conceived one for RSM, with the difference that the 262 classic modulated symbols of all RAs have to be detected, as 263 opposed to those of $N_{a}$ antennas only for RSM. Accordingly, 264 the receiver first detects the set of antennas with the highest 265 received power levels and then detects the classic modulated 266 symbols at all RAs according to

$$
\hat{k}=\arg \max _{j \in \mathcal{J}} \sum_{i=1}^{N_{a}}\left|y_{j, i}\right|^{2}
$$

267 where $\mathcal{J}$ denotes the set of symbols in the spatial domain, and

$$
\hat{\mathbf{b}}_{m}=\arg \min _{n \in \mathcal{Q}}\left|\mathbf{y} / f-\mathbf{b}_{n}\right|^{2}
$$

268 where $\mathcal{Q}$ denotes the classic modulation constellation, and $b_{n}$ 269 are the symbols in the modulated symbol alphabet.
TABLE I

BE IN BITS PER CHANNEL USE FOR SMX, RSM, AND DLT

\begin{tabular}{|l||r|}
\hline & Bandwidth Efficiency (BE) \\
\hline \hline SMX & $\beta=N_{r} \log _{2}(M)$ \\
\hline RSM & $\beta=N_{a} \log _{2}(M)+\log _{2}\left(\begin{array}{c}N_{r} \\
N_{a}\end{array}\right)$ \\
\hline DLT & $\beta=N_{r} \log _{2}(M)+\log _{2}\left(\begin{array}{c}N_{r} \\
N_{a}\end{array}\right)$ \\
\hline
\end{tabular}

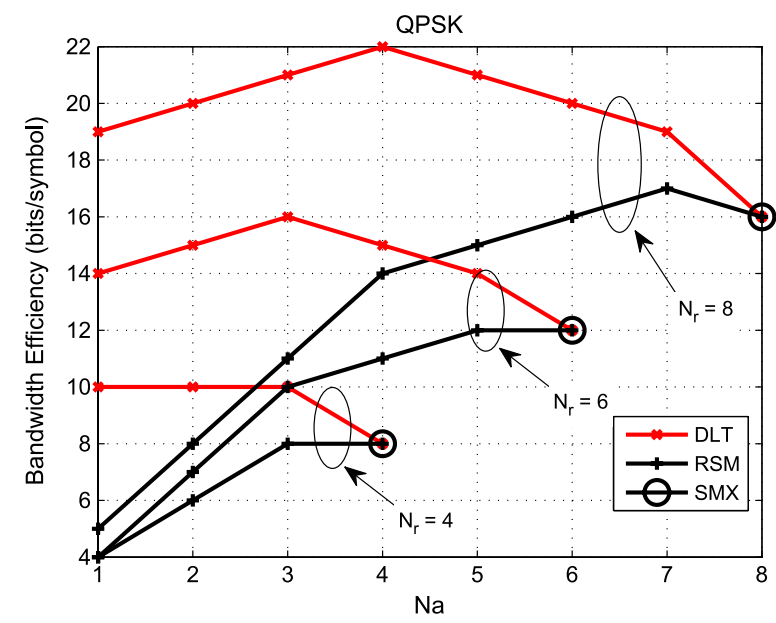

Fig. 2. BE versus $N_{a}$ for SMX, RSM, and DLT using the expressions of Table I.

\section{A. Bandwidth Efficiency}

Clearly, the encoding process in (8) and (9) encodes 271 $N_{r} \log _{2}(M)$ bits in the modulated symbol domain and an 272 additional $\log _{2}\left(\begin{array}{l}N_{r} \\ N_{a}\end{array}\right)$ bits in the spatial domain. This results in a 273 total of

$$
\beta=N_{r} \log _{2}(M)+\log _{2}\left(\begin{array}{l}
N_{r} \\
N_{a}
\end{array}\right)
$$

bits per transmitted supersymbol for DLT, which is strictly 275 greater than that for SMX and RSM. Here, the notation $N_{a} 276$ denotes the number of antennas receiving symbols at the power 277 level $P_{1}$. We should emphasize that, although all RAs are active 278 for both RSM and the proposed DLT, for coherence with the SM 279 literature, we shall adhere to the terms "active" and "inactive" 280 to indicate the antennas receiving $\{1,0\}$ and $\left\{P_{1}, P_{2}\right\}$ for RSM 281 and DLT, respectively. A comparison of the BEs of SMX, RSM, 282 and DLT is shown in Table I, where it can be seen that the 283 proposed DLT approach has an improved BE compared with 284 the conventional approaches. This is quantified in Fig. 2, where 285 the $\mathrm{BE}$ is expressed in terms of bits per channel use is shown 286 with increasing numbers of "active" antennas $N_{a}$ for MIMO 287 links with $N_{r}=4, N_{r}=6$, and $N_{r}=8$, where the clear bene- 288 fits of the proposed approach can be seen. It can be observed 289 that the additional BE of DLT compared with SMX can be 290 maximized by appropriately selecting the number of activated 291 antennas according to

$$
\tilde{N}_{a}=\arg \max _{N_{a}} \log _{2}\left(\begin{array}{c}
N_{r} \\
N_{a}
\end{array}\right)=N_{r} / 2
$$

which is demonstrated in the figure. 


\section{B. Symbol Power Levels}

295 With regard to the resulting BER performance, the set of 296 spatial power levels $\left\{P_{1}, P_{2}\right\}$ must be carefully selected so that 297 they satisfy a combination of two constraints.

299 1) There is sufficient separation between the two power lev300 els $P_{1}, P_{2}$ for correct detection of the "active" antennas $301 \quad$ and hence the spatial symbol $k$ in the presence of noise.

302 2) The symbols received with $P_{2}<P_{1}$ that dominate the 303 BER of the modulated symbol detection must experience 304 a sufficiently high signal-to-noise ratio (SNR) that is 305 adequate for reliable demodulation.

306 Let us therefore define the power ratio

$$
\alpha=\frac{P_{2}}{P_{1}}
$$

307 as the ratio between the two power levels transmitted, which 308 is optimized in the following results. Since $N_{a}$ symbols are 309 transmitted with power $P_{1}$ and the remaining $N_{r}-N_{a}$ symbols 310 have power of $P_{2}$, given a total power budget of $P=1$, we have

$$
\begin{aligned}
& P_{1}=\frac{1}{\left(N_{r}-N_{a}\right) \alpha+N_{a}} \\
& P_{2}=\frac{\alpha}{\left(N_{r}-N_{a}\right) \alpha+N_{a}} .
\end{aligned}
$$

311 Clearly, since the power levels $P_{1}, P_{2}$ influence the reliability 312 of detection for the modulated symbols and since the ratio $\alpha$ 313 determines the detection reliability of the spatial symbols, $\alpha$ 314 can be optimized for best BER performance. In the following, 315 we derive a closed-form expression for the optimum $\alpha$ value 316 for an $M$-order PSK modulation, where it can be seen that this 317 optimum value is independent of both $N_{r}$ and of $N_{a}$.

318 Remark: Regarding the effect of the above on the transmit 319 power distribution, we note that the power imbalance discussed 320 refers to the information symbols $\mathbf{s}_{m}^{k}$ and does not translate 321 to a power imbalance for the transmit symbols $\mathbf{t}$. Indeed, the 322 ZF-precoded transmit symbols have the same average transmit 323 power, constrained by the scaling factor $f$ as shown above, 324 which is valid for both the proposed DLT and for the conven325 tional SMX, and these transmit symbols exhibit the same power 326 distribution for both techniques. In other words, the proposed 327 scheme does not impact the design of the power amplifiers used 328 at the transmitter.

329 To verify the above, Fig. 3 shows the probability density 330 function (pdf) of the normalized transmit power per antenna 331 for both SMX and DLT in a $(8 \times 4)$ element MIMO system. It 332 can be seen that, as expected, both techniques show the same 333 distribution of transmit power. \\ IV. Dual-Layered Transmission Performance ANALYSIS AND OPTIMUM POWER RATIO $\alpha$}

\section{A. Probability of Error}

337 Here, we carry out a performance analysis for the proposed 338 DLT scheme by deriving the PEP between the pair of symbols

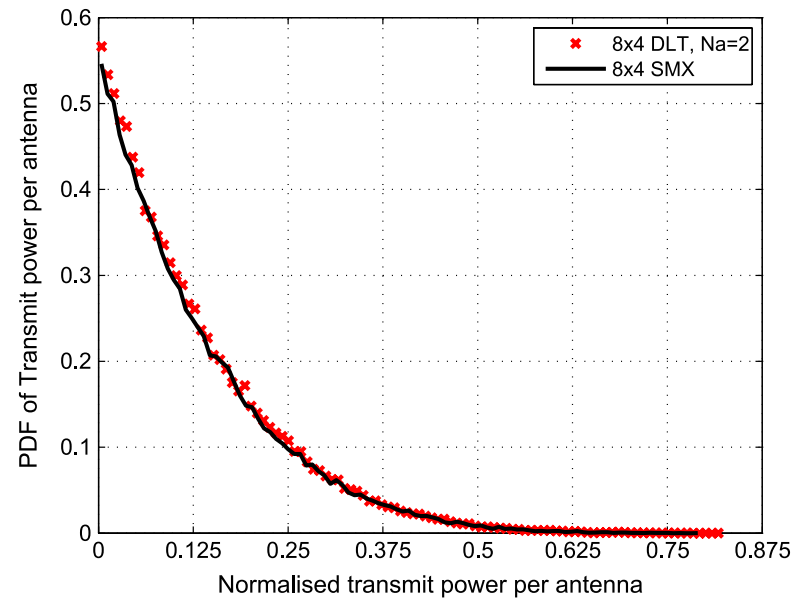

Fig. 3. PDF of transmit power per antenna for a $(8 \times 4)$ MIMO with SMX and DLT and QPSK with Rayleigh fading.

$\mathbf{s}_{m}^{k}$ and $\mathbf{s}_{n}^{l}$ in the superimposed spatial and classic modulation 339 constellations, following the analysis in [36]. Accordingly, we 340 define the PEP as $\mathcal{P}\left(\mathbf{s}_{m}^{k} \rightarrow \mathbf{s}_{n}^{l}\right)$ and use the union bound for the 341 average bit error probability $P_{e}$, which is expressed as

$$
P_{e} \leq \frac{1}{\beta} E\left\{\sum_{\mathbf{s}_{m}^{k} \in \mathcal{B}} \sum_{\mathbf{s}_{n}^{l} \in \mathcal{B} \neq \mathbf{s}_{m}^{k}} d\left(\mathbf{s}_{m}^{k}, \mathbf{s}_{n}^{l}\right) \mathcal{P}\left(\mathbf{s}_{m}^{k} \rightarrow \mathbf{s}_{n}^{l}\right)\right\}
$$

where $d\left(\mathbf{s}_{m}^{k}, \mathbf{s}_{n}^{l}\right)$ is the Hamming distance between the bit 343 representations of symbols $\mathbf{s}_{m}^{k}, \mathbf{s}_{n}^{l}$ and $\mathcal{B}=\mathcal{J} \cup \mathcal{Q}$ is the super- 344 symbol constellation defined as the union of the spatial domain 345 constellation and the classic modulation constellation. We have 346 used the operator $\cup$ to define the union of sets. For the PEP, we 347 have the following theorem.

348

Theorem 1: The PEP $\mathcal{P}\left(\mathbf{s}_{m}^{k} \rightarrow \mathbf{s}_{n}^{l}\right)$ for DLT can be expressed as 349

$\mathcal{P}\left(\mathbf{s}_{m}^{k} \rightarrow \mathbf{s}_{n}^{l}\right)=Q\left(\frac{f}{\sqrt{N_{0}}}\left(1-\sum_{i=1}^{N_{r}} \sqrt{p_{k_{i}} p_{l_{i}}} \mathcal{R}\left\{b_{m_{i}}^{*} b_{n_{i}}\right\}\right)\right)$

where $Q(\cdot)$ denotes the Gaussian $q$-function [42], $\mathcal{R}\{\cdot\}$ denotes 350 the real part of a number, $(\cdot)^{*}$ denotes the complex conjugate 351 operation, and $N_{0}=2 \sigma^{2}$ is the noise power spectral density. $\quad 352$

Proof: Let us first define $\mathbf{r}=\mathbf{y} / f$ and $\mathbf{v}=\mathbf{w} / f$ for 353 use in the following expressions. The PEP of the supersymbol 354 constellation can be expressed as

$$
\begin{aligned}
\mathcal{P}\left(\mathbf{s}_{m}^{k} \rightarrow \mathbf{s}_{n}^{l}\right)=\mathcal{P} & \left(\left\|\mathbf{r}-\mathbf{s}_{m}^{k}\right\|^{2}>\left\|\mathbf{r}-\mathbf{s}_{n}^{l}\right\|^{2}\right) \\
=\mathcal{P}( & \left(\sum_{i=1}^{N_{r}} p_{k_{i}}\left|b_{m_{i}}\right|^{2}-2 \mathcal{R}\left\{r_{i}^{*} \sqrt{p_{k_{i}}} b_{m_{i}}\right\}\right. \\
& \left.>\sum_{i=1}^{N_{r}} p_{l_{i}}\left|b_{n_{i}}\right|^{2}-2 \mathcal{R}\left\{r_{i}^{*} \sqrt{p_{l_{i}}} b_{n_{i}}\right\}\right) .
\end{aligned}
$$

Since, for PSK signals, we have $\left|b_{m_{i}}\right|=1$, by rearranging 356 the terms in the probability expression, (21) can be further 357 
358 simplified as

$$
\begin{gathered}
\mathcal{P}\left(\mathbf{s}_{m}^{k} \rightarrow \mathbf{s}_{n}^{l}\right)=\mathcal{P}\left(\sum_{i=1}^{N_{r}} \mathcal{R}\left\{r_{i}^{*} \sqrt{p_{l_{i}}} b_{n_{i}}\right\}-\mathcal{R}\left\{r_{i}^{*} \sqrt{p_{k_{i}}} b_{m_{i}}\right\}\right. \\
\left.>\frac{\sum_{i=1}^{N_{r}} p_{l_{i}}-\sum_{i=1}^{N_{r}} p_{k_{i}}}{2}\right) \cdot
\end{gathered}
$$

359 Since $\sum_{i=1}^{N_{r}} p_{l_{i}}=\sum_{i=1}^{N_{r}} p_{k_{i}}=1$ and $r_{i}=\sqrt{p_{k_{i}}} b_{m_{i}}+v_{i}$, we 360 have

$$
\begin{gathered}
\mathcal{P}\left(\mathbf{s}_{m}^{k} \rightarrow \mathbf{s}_{n}^{l}\right)=\mathcal{P}\left(\sum_{i=1}^{N_{r}} \mathcal{R}\left\{\sqrt{p_{k_{i}}} b_{m_{i}}^{*} \sqrt{p_{l_{i}}} b_{n_{i}}\right\}+\mathcal{R}\left\{v_{i}^{*} \sqrt{p_{l_{i}}} b_{n_{i}}\right\}\right. \\
\left.>\sum_{i=1}^{N_{r}} p_{k_{i}}\left|b_{m_{i}}\right|^{2}+\mathcal{R}\left\{v_{i}^{*} \sqrt{p_{k_{i}}} b_{m_{i}}\right\}\right) \\
=\mathcal{P}\left(\sum_{i=1}^{N_{r}} \mathcal{R}\left\{v_{i}^{*}\left(\sqrt{p_{l_{i}}} b_{n_{i}}-\sqrt{p_{k_{i}}} b_{m_{i}}\right)\right\}\right. \\
\left.>1-\sum_{i=1}^{N_{r}} \sqrt{p_{k_{i}} p_{l_{i}}} \mathcal{R}\left\{b_{m_{i}}^{*} b_{n_{i}}\right\}\right) .
\end{gathered}
$$

361 Let us define the random variable $\chi \hat{=} \sum_{i=1}^{N_{r}} \mathcal{R}\left\{v_{i}^{*}\left(\sqrt{p_{l_{i}}} b_{n_{i}}-\right.\right.$ $\left.\left.362 \sqrt{p_{k_{i}}} b_{m_{i}}\right)\right\}$ for which we have $\chi \in \mathcal{N}\left(0, A N_{0} / f^{2}\right)$ with

$$
A=\frac{\sum_{i=1}^{N_{r}} p_{l_{i}}\left|b_{n_{i}}\right|^{2}+p_{k_{i}}\left|b_{n_{i}}\right|^{2}}{2}=\frac{1}{2} \sum_{i=1}^{N_{r}} p_{l_{i}}+p_{k_{i}}
$$

363 For the unity transmit power assumed in this paper, it can be 364 seen from (24) that $A=1$. Accordingly, for the PEP, we have

$$
\mathcal{P}\left(\mathbf{s}_{m}^{k} \rightarrow \mathbf{s}_{n}^{l}\right)=\mathcal{P}\left(\chi>1-\sum_{i=1}^{N_{r}} \sqrt{p_{k_{i}} p_{l_{i}}} \mathcal{R}\left\{b_{m_{i}}^{*} b_{n_{i}}\right\}\right)
$$

365 which, for $\chi \in \mathcal{N}\left(0, N_{0} / f^{2}\right)$, leads to (20).

\section{B. Optimum Power Ratio $\alpha$}

367 As mentioned earlier, the power ratio $\alpha$ determines the 368 reliability of detection for the spatial symbol, whereas the lower 369 power level $P_{2}$ dominates the BER performance of the classic 370 modulated symbols' detection. As the probability of error in 371 (19) is dominated by the maximum PEP, the optimum power 372 ratio should be selected as

$$
\alpha_{\mathrm{opt}}=\arg \min _{\alpha} \max _{\mathbf{s}_{m}^{k}, \mathbf{s}_{n}^{l}}\left\{\mathcal{P}\left(\mathbf{s}_{m}^{k} \rightarrow \mathbf{s}_{n}^{l}\right)\right\}
$$

373 To simplify the analysis, we shall treat the errors in the spatial 374 and classic modulated symbols separately. Accordingly, for the 375 maximum PEP $\mathcal{P}_{m}\left(s_{m_{i}}^{k} \rightarrow s_{m_{i}}^{l}\right)$ in the spatial domain only, we 376 have the following theorem.
Theorem 2: The maximum PEP $\mathcal{P}_{m}\left(s_{m_{i}}^{k} \rightarrow s_{m_{i}}^{l}\right)$ for the 377 spatial symbols in DLT can be expressed as

$$
\mathcal{P}_{m}\left(s_{m_{i}}^{k} \rightarrow s_{m_{i}}^{l}\right)=Q\left(\frac{f}{\sqrt{N_{0}}} \cdot \frac{\sqrt{P_{2}}-\sqrt{P_{1}}}{2}\right) .
$$

Proof: The maximum PEP in the spatial domain involves 379 the adjacent symbols of different power levels in the supersym- 380 bol constellation and can be expressed as

$$
\begin{aligned}
\mathcal{P}_{m} & \left(s_{m_{i}}^{k} \rightarrow s_{m_{i}}^{l}\right) \\
& =\mathcal{P}\left(\left\|r_{i}-s_{m_{i}}^{k}\right\|^{2}>\left\|r_{i}-s_{m_{i}}^{l}\right\|^{2}\right) \\
& =\mathcal{P}\left(P_{1}-2 \mathcal{R}\left\{r_{i}^{*} \sqrt{P_{1}} b_{m_{i}}\right\}>P_{2}-2 \mathcal{R}\left\{r_{i}^{*} \sqrt{P_{2}} b_{m_{i}}\right\}\right)
\end{aligned}
$$

where, using $r_{i}=\sqrt{p_{k_{i}}} b_{m_{i}}+v_{i}$, we get

$$
\begin{aligned}
\mathcal{P}_{m}\left(s_{m_{i}}^{k} \rightarrow s_{m_{i}}^{l}\right) & \\
= & \mathcal{P}\left(P_{1}-2 P_{1}\left|b_{m_{i}}\right|^{2}-2 \mathcal{R}\left\{u_{i}^{*} \sqrt{P_{1}} b_{m_{i}}\right\}\right. \\
& \left.>P_{2}-2 \sqrt{P_{1} P_{2}}\left|b_{m_{i}}\right|^{2}-2 \mathcal{R}\left\{u_{i}^{*} \sqrt{P_{2}} b_{m_{i}}\right\}\right) \\
= & \mathcal{P}\left(2\left(\sqrt{P_{2}}-\sqrt{P_{1}}\right) \mathcal{R}\left\{u_{i}^{*} b_{m_{i}}\right\}>P_{1}+P_{2}-2 \sqrt{P_{1} P_{2}}\right) \\
= & \mathcal{P}\left(-\mathcal{R}\left\{u_{i}^{*} b_{m_{i}}\right\}>\frac{\sqrt{P_{1}}-\sqrt{P_{2}}}{2}\right) .
\end{aligned}
$$

Similarly to the given proof, we have used the fact 383 that $\left|b_{m_{i}}\right|^{2}=1$, and it can be seen that $\psi \hat{=}-\mathcal{R}\left\{u_{i}^{*} b_{m_{i}}\right\} \in 384$ $\mathcal{N}\left(0, N_{0} / f^{2}\right)$. Accordingly, for the minimum PEP in the spatial 385 constellation, we have

$$
\mathcal{P}_{m}\left(s_{m_{i}}^{k} \rightarrow s_{m_{i}}^{l}\right)=\mathcal{P}\left(\psi>\frac{\sqrt{P_{2}}-\sqrt{P_{1}}}{2}\right)
$$

which leads to (27).

This indicates that the separation between $\left\{P_{1}, P_{2}\right\}$ should 388 be maximized for minimizing the errors in the spatial bits, 389 which are dominated by the distance between the pairs of adja- 390 cent symbols having different power levels $d_{s}=\sqrt{P_{1}}-\sqrt{P_{2}} \cdot 391$ We therefore define the spatial function $f_{S}(\alpha)$ that accounts for 392 the dependence of the spatial errors on $\alpha$ as

$$
f_{S}(\alpha) \triangleq \sqrt{P_{1}}-\sqrt{P_{2}}=\frac{1-\sqrt{\alpha}}{\sqrt{\left(N_{r}-N_{a}\right) \alpha+N_{a}}} .
$$

As regards to the classic modulated symbol errors, it is 394 known that the PSK error probability is given as [41]

$$
\begin{aligned}
\mathcal{P}\left(s_{m_{i}}^{k} \rightarrow s_{n_{i}}^{k}\right) & =\mathcal{P}\left(\left\|r_{i}-s_{m_{i}}^{k}\right\|^{2}>\left\|r_{i}-s_{n_{i}}^{l}\right\|^{2}\right) \\
& =Q\left(f \sqrt{\frac{P_{2}}{N_{0}} \log _{2}(M) \sin \frac{\pi}{M}}\right) .
\end{aligned}
$$




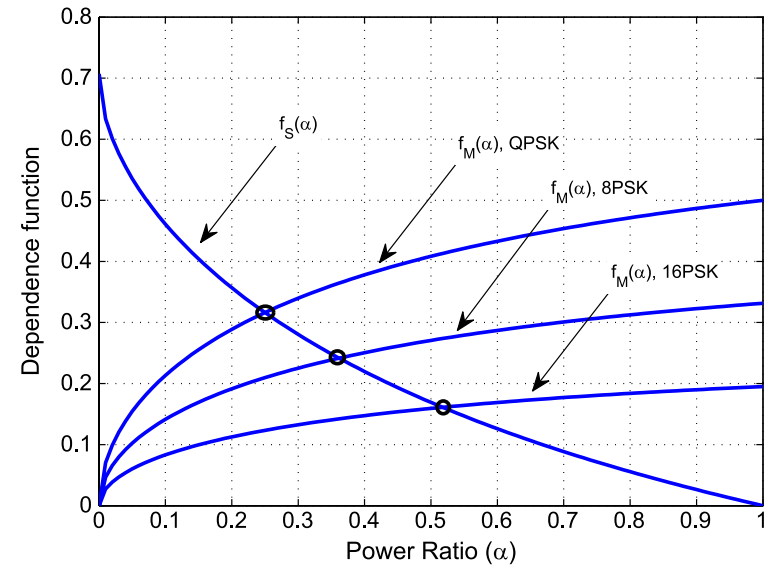

Fig. 4. Theoretical optimization of $\alpha$ for DLT for a $(8 \times 4)$ MIMO with $N_{a}=2$, using (36).

396 Accordingly, we define the function $f_{M}(\alpha)$ for the dependence 397 of the modulated symbol error on $\alpha$ as

$$
\begin{aligned}
f_{M}(\alpha) & \triangleq \sqrt{P_{2} \log _{2}(M) \sin \frac{\pi}{M}} \\
& =\sqrt{\log _{2}(M) \sin \frac{\pi}{M} \cdot \frac{\alpha}{\left(N_{r}-N_{a}\right) \alpha+N_{a}}} .
\end{aligned}
$$

398 The optimization (26) is equivalent to the maximization of 399 the minimum of these functions:

$$
\alpha_{\mathrm{opt}}=\arg \max _{\alpha}\left\{\min \left\{f_{S}(\alpha), f_{M}(\alpha)\right\}\right\} .
$$

400 The optimum power scaling ratio is, therefore, given as

$$
\begin{array}{r}
\alpha_{\mathrm{opt}}=\arg \max _{\alpha}\left\{\frac{1-\sqrt{\alpha}}{\sqrt{\left(N_{r}-N_{a}\right) \alpha+N_{a}}},\right. \\
\left.\sqrt{\log _{2}(M) \sin \frac{\pi}{M} \cdot \frac{\alpha}{\left(N_{r}-N_{a}\right) \alpha+N_{a}}}\right\}
\end{array}
$$

401 which is equivalent to selecting the factor $\alpha$ so that the two 402 terms in the minimization become equal, which gives

$$
\alpha_{\mathrm{opt}}=\frac{1}{\left(1+\sqrt{\log _{2}(M) \sin \frac{\pi}{M}}\right)^{2}} .
$$

403 We examine this optimization in Fig. 4, which shows the 404 functions $f_{s}(\alpha), f_{M}(\alpha)$ when increasing the values of $\alpha$ for 405 the example of a $(8 \times 4)$-element DLT system with $N_{a}=2$, 406 for $M=4,8,16$, i.e., quadrature phase-shift keying (QPSK), 407 8PSK, and 16PSK modulation. The intersections of the lines 408 determine the optimum values of $\alpha$. It will be shown in the 409 following that the theoretically obtained optimal values of $\alpha$ 410 closely match the optimal values obtained by simulation.

\section{1 \\ V. COMPleXity AND POWER EFFICIENCY}

\section{A. Complexity}

413 Here, we compare the computational complexity of SMX, 414 RSM, and DLT and use this to carry out a comparison of the 415 resulting PE of the techniques. First, Table II summarizes the
TABLE II

COMPLEXITY FOR SMX, RSM, AND THE PROPOSED DLT SCHEME. $N_{\chi}=N_{a}$ FOR RSM, $N_{\chi}=N_{r}$ FOR DLT

\begin{tabular}{|l|r|}
\hline & Operations \\
\hline \hline \multicolumn{2}{|l|}{ Transmitter: } \\
\hline ZF processing & $N_{r}^{3}+2 N_{t} N_{r}$ \\
\hline Receiver: \\
\hline Spatial detection & $2 N_{a}\left(\begin{array}{l}N_{r} \\
N_{a}\end{array}\right)$ \\
\hline Demodulation & $N_{\chi} M$ \\
\hline \hline SMX Total & $C_{S M X}=N_{r}^{3}+N_{r}\left(2 N_{t}+M\right)$ \\
\hline RSM Total & $C_{R S M}=N_{r}^{3}+2 N_{t} N_{r}+N_{a}\left(2\left(\begin{array}{c}N_{r} \\
N_{a}\end{array}\right)+M\right)$ \\
\hline DLT Total & $C_{D L T}=N_{r}^{3}+N_{r}\left(2 N_{t}+M\right)+2 N_{a}\left(\begin{array}{l}N_{r} \\
N_{a}\end{array}\right)$ \\
\hline
\end{tabular}

computational complexity of each of the techniques, taking into 416 account the dominant operations at the transmitter and receiver. 417 We follow the typical assumption that multiplications and ad- 418 ditions require an equal number of floating point operations. 419 For all three schemes, the ZF-TPC employed at the transmitter 420 involves the inversion of the channel matrix that requires $N_{r}^{3}+421$ $N_{t} N_{r}$ operations and the multiplication with the supersymbol 422 vector involving an additional $N_{t} N_{r}$ operations. At the receiver, 423 all techniques require a demodulation stage that involves $M 424$ comparisons for and $M$-order modulation, for each antenna 425 receiving information, i.e., $N_{r} M$ for both SMX and DLT, and 426 $N_{a} M$ for RSM. The RSM and DLT require an additional stage 427 for the detection of the spatial symbol which, from (6) involves 428 $N_{a}$ complex multiplications and $N_{a}$ complex additions for each 429 antenna combination out of the $\left(\begin{array}{l}N_{r} \\ N_{a}\end{array}\right)$ combinations in total. 430

\section{B. Power Efficiency}

As the ultimate metric for evaluating the performance- 432 complexity tradeoff and the overall usefulness of the proposed 433 technique, we consider the PE of DLT compared with SMX and 434 RSM. Following the modeling of [43]-[46], we define the PE of 435 the communication link as the bit rate per total transmit power 436 dissipated, i.e., the ratio of the goodput achieved over the power 437 consumed:

$$
\mathcal{E}=\frac{T}{P_{\mathrm{PA}}+N_{t} \cdot P_{t}^{\mathrm{RF}}+N_{r} \cdot P_{r}^{\mathrm{RF}}+p_{c} \cdot C}
$$

where $P_{\mathrm{PA}}=((\xi / \eta)-1) P$ in Watts is the power dissipated by 439 the power amplifier to produce the total transmit signal power 440 $P$, with $\eta$ being the power amplifier's efficiency and $\xi$ being the 441 modulation-dependent peak-to-average power ratio (PAPR). 442 Furthermore, $P_{t}^{\mathrm{RF}}=P_{\text {mix }}+P_{\text {filt }}+P_{\mathrm{DAC}}$ and $P_{r}^{\mathrm{RF}}=P_{\text {mix }}+443$ $P_{\text {filt }}+P_{\mathrm{ADC}}$ represent the RF powers related to the mix- 444 ers, to the transmit filters, to the digital-to-analog con- 445 verter (DAC) at the transmitter and to the analog-to-digital 446 converter (ADC) at the receiver, which are assumed to 447 be constant for the purposes of this paper. We use prac- 448 tical values of these from [44] as $\eta=0.35$ and $P_{\text {mix }}=449$ $30.3 \mathrm{~mW}, P_{\text {filt }}=2.5 \mathrm{~mW}, P_{\mathrm{DAC}}=1.6 \mathrm{~mW}$, and $P_{\mathrm{DAC}}=450$ $1.3 \mathrm{~mW}$, yielding $P_{t}^{\mathrm{RF}}=34.4 \mathrm{~mW}$, and $P_{r}^{\mathrm{RF}}=34.1 \mathrm{~mW} .451$ In (37), $p_{c}$ in Watts/KOps is the power per $10^{3}$ elementary 452 
453 operations (KOps) of the digital signal processor, and $C$ is the 454 number of operations involved, taken from Table II, where 455 it is assumed that the operations shown dominate the digital 456 signal processing complexity of the link. This term is used 457 for introducing the complexity as a factor related to the power 458 dissipation in the PE metric. Typical values of $p_{c}$ include $p_{c}=$ $45922.88 \mathrm{~mW} / \mathrm{KOps}$ for the Virtex-4 and $p_{c}=5.76 \mathrm{~mW} / \mathrm{KOps}$ for 460 the Virtex-5 FPGA family from Xilinx [47]. Finally

$$
T=\beta B\left(1-P_{B}\right)=\beta B\left(1-P_{e}\right)^{B}
$$

461 represents the achieved goodput, where $P_{B}$ is the block error 462 rate with a block of size $B$ symbols, and $\beta$ is the BE of SM 463 in bits per symbol, taken from Table I. For reference, we have 464 assumed an LTE Type-2 TDD frame structure [48]. This has 465 a 10 ms duration that consists of 10 subframes, out of which 466 five subframes, containing 14 symbol time slots each, are used 467 for DL transmission yielding a block size of $B=70$ for the $468 \mathrm{DL}$, whereas the remainder are used for both uplink (UL) and 469 control information transmission. A slow fading channel is 470 assumed where the channel remains constant for the duration 471 of the frame.

472 The expression in (37) provides an amalgamated metric that 473 combines goodput, complexity, and transmit signal power, all 474 in a unified metric. High values of $\mathcal{E}$ indicate that high bit 475 rates are achievable for a given power consumption and thus 476 denote high energy efficiency. The following results show that 477 DLT provides an increased energy efficiency compared with 478 SMX and RSM in numerous scenarios using different transmit 479 power levels $P$.

\section{0 \\ VI. Numerical Results}

481 To evaluate the benefits of the proposed technique, this 482 section presents numerical results based on Monte Carlo sim483 ulations of SMX, RSM, and the proposed DLT. The channel 484 impulse response is assumed perfectly known at the transmitter. 485 Without loss of generality, unless stated otherwise, we assume 486 that the transmit power is restricted to $P=1$. MIMO systems 487 with up to eight TAs employing QPSK and 8PSK modulation 488 are explored, albeit it is plausible that the benefits of the 489 proposed technique extend to larger scale systems and higher 490 order modulation.

491 Remark: It should be noted that the BE improvement shown 492 in the following could also be obtained by SMX with the aid 493 of an increased classical modulation order. Accordingly, in the 494 following, we compare the proposed DLT to: (a) SMX using the 495 same classical modulation order to illustrate the improved BE 496 of DLT; and (b) SMX relying on a higher modulation order to 497 highlight the improved performance of DLT for an identical BE. 498 In Fig. 5, we show the BER as a function of the power 499 ratio for DLT for the $(8 \times 4)$ MIMO system, where the values 500 of $\alpha$ in the area of 0.25 can be seen to provide the best 501 performance. This matches well with the theoretically derived 502 result of Section IV-A and Fig. 4. Similarly, Fig. 6 shows the 503 BER versus $\alpha$ performance for higher order modulation 8PSK 504 and 16PSK. Again, a close match can be seen with the theo505 retically derived values for $\alpha_{\text {opt }}$. In Fig. 7, we show the BER 506 with increasing SNR for the proposed DLT, where the black

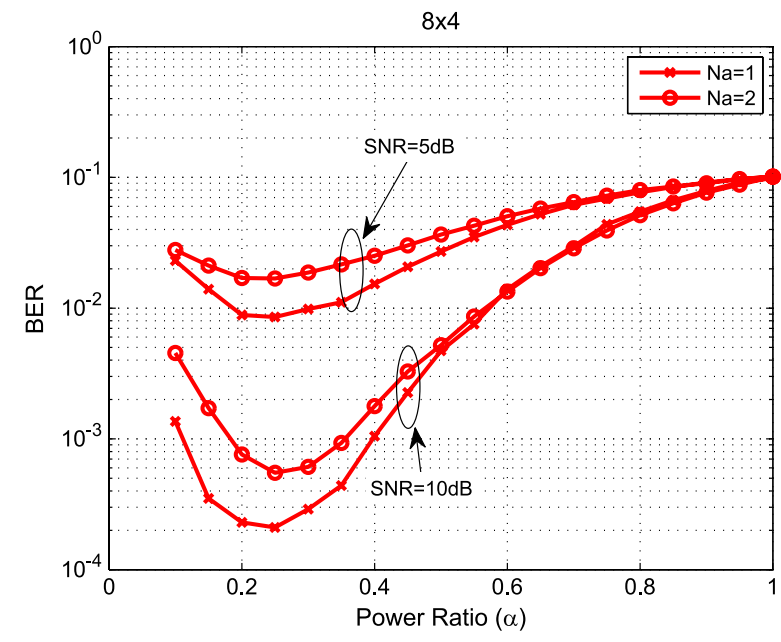

Fig. 5. BER versus $\alpha$ for an $(8 \times 4)$ MIMO with SMX and DLT, as well as QPSK with Rayleigh fading.
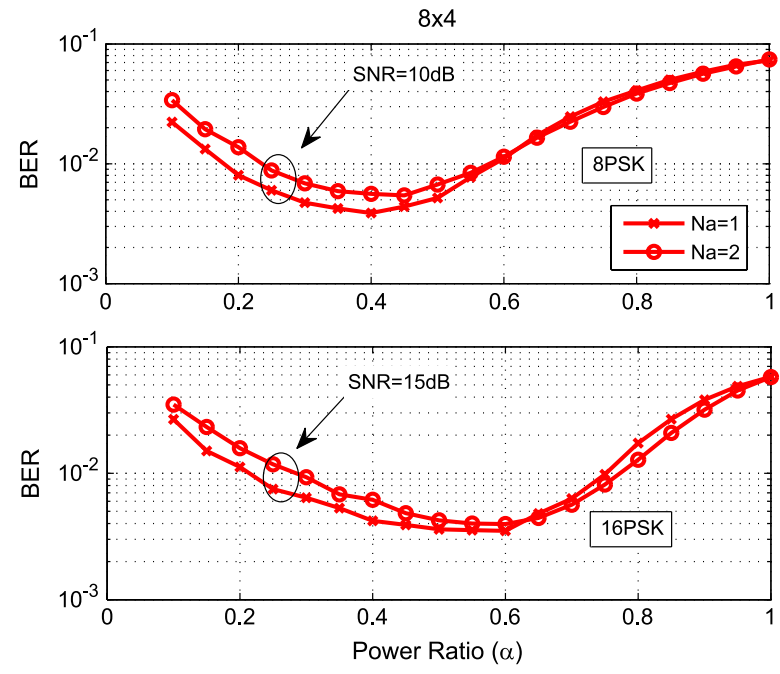

Fig. 6. BER versus $\alpha$ for a $(8 \times 4)$ MIMO with DLT, as well as 8 PSK and 16PSK with Rayleigh fading.

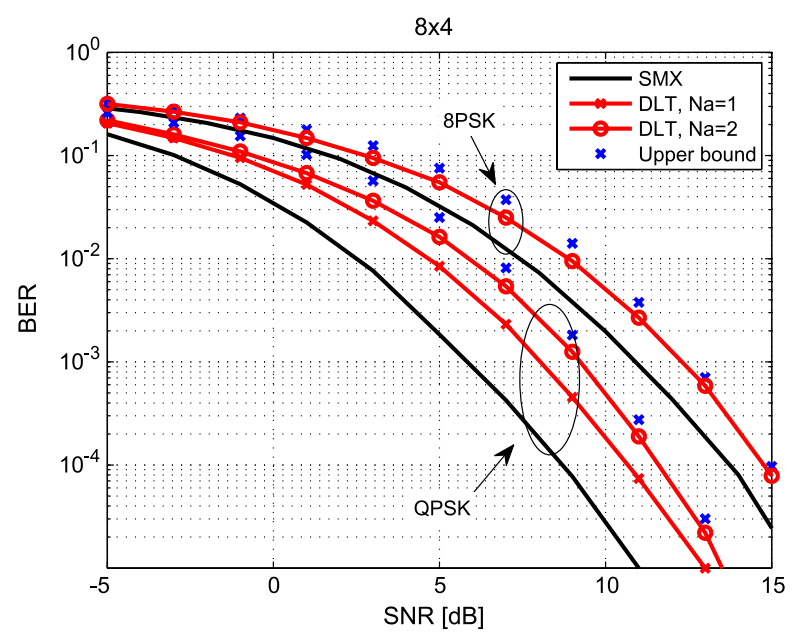

Fig. 7. BER versus SNR for a $(8 \times 4)$ MIMO with SMX and DLT, as well as QPSK and 8PSK with Rayleigh fading. 


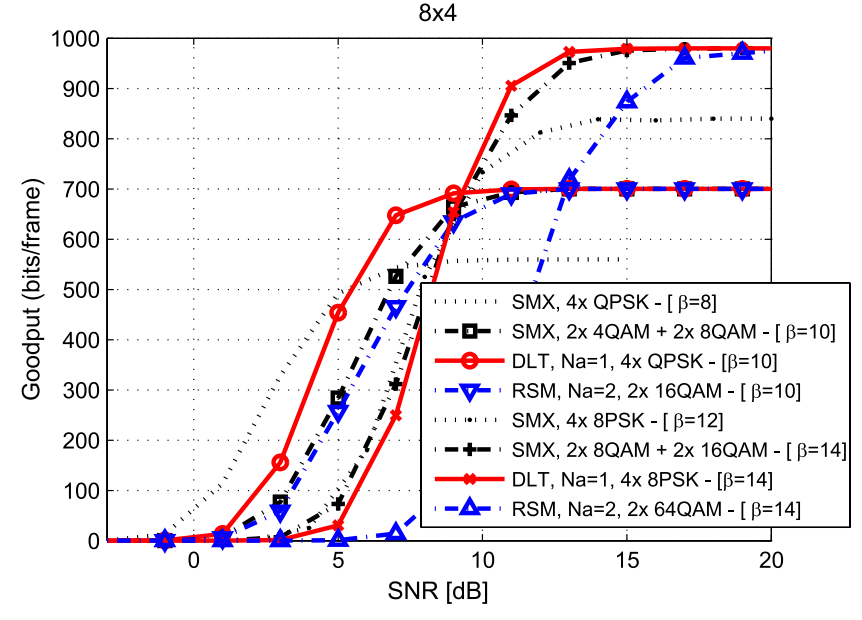

Fig. 8. Goodput versus SNR for a $(8 \times 4)$ MIMO with SMX and DLT, as well as Rayleigh fading.

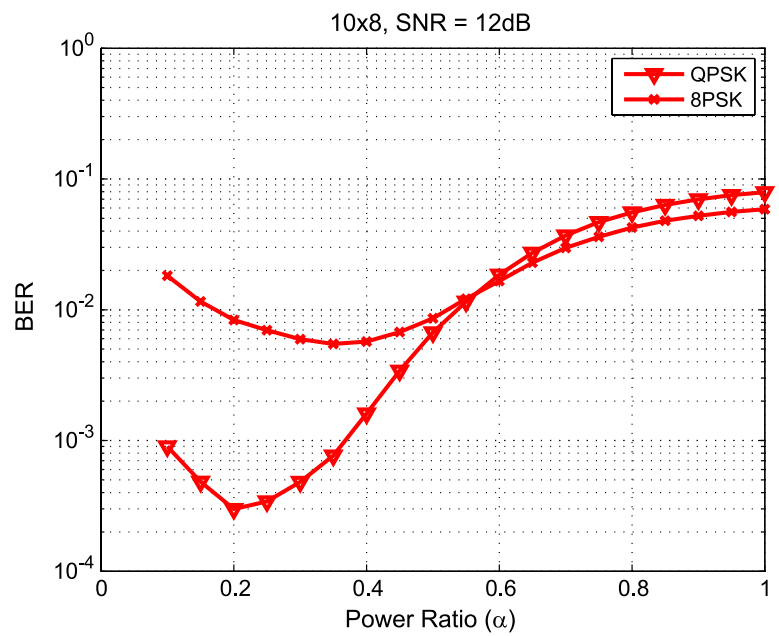

Fig. 9. BER versus $\alpha$ for a $(10 \times 8)$ MIMO with DLT, QPSK, and 8PSK with Rayleigh fading.

507 lines for $N_{a}=4$ represent SMX transmission. The curves show 508 results for both QPSK and 8PSK. The theoretical upper bound 509 using (19) is also depicted for both cases, and it can be observed 510 that it offers a tight bound. Clearly, the DLT scheme has inferior 511 BER performance compared with SMX due to the additional 512 spatial streams but at the benefit of improved BE. The improved $513 \mathrm{BE}$ of DLT is demonstrated in Fig. 8 where the goodput 514 with increasing SNR is depicted for the same $(8 \times 4)$ MIMO 515 scenario. Clearly, DLT provides higher goodput than SMX for 516 sufficiently high SNR values. To complete our comparisons, for 517 both scenarios in the figure, we also show the cases where the 518 symbol modulation order used for SMX and RSM is increased 519 for some of the spatial streams in order to achieve the same $520 \mathrm{BE}$ values of $\beta=10$ and $\beta=14$ with the proposed DLT, for 521 QPSK and 8PSK, respectively. Clearly, this has an impact on 522 the SNR requirement of SMX, where it can be seen that the 523 proposed DLT scheme obtains the maximum goodput at lower 524 SNR values.

525 The performance comparison is extended to the $(10 \times 8)$ 526 MIMO system in Figs. 9 and 10. In Fig. 9, we show the

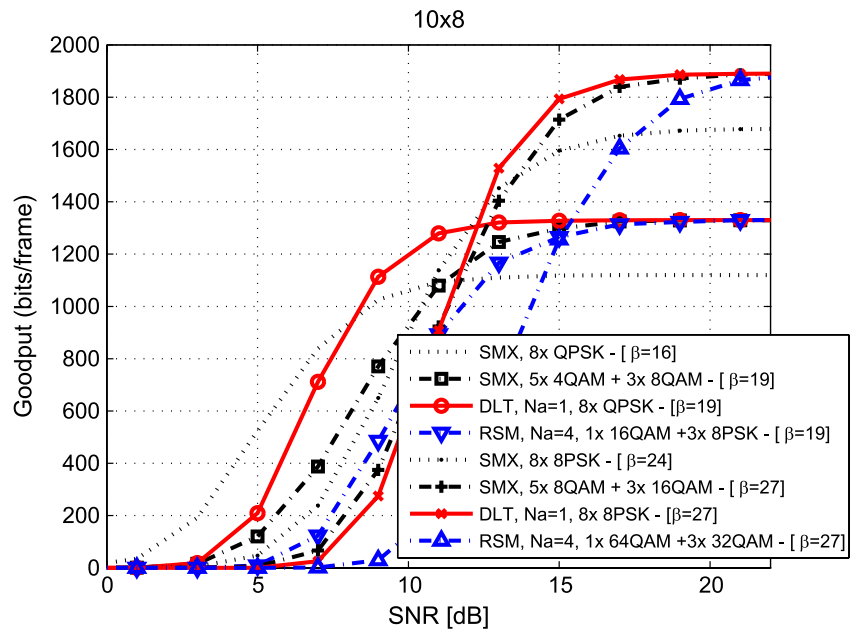

Fig. 10. Goodput versus SNR for a $(10 \times 8)$ MIMO with SMX and DLT, as well as Rayleigh fading.

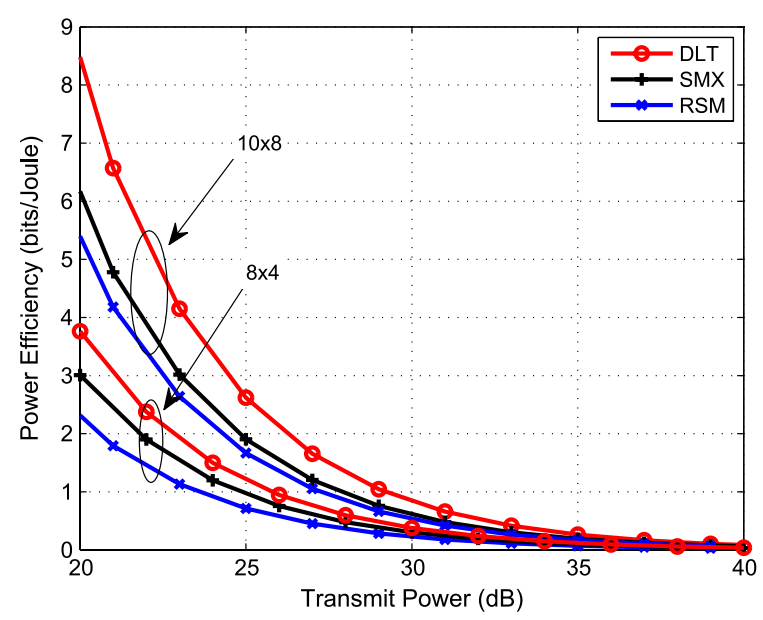

Fig. 11. PE versus transmit power for $(10 \times 8)$ and $(8 \times 4)$ MIMO systems with SMX and DLT, as well as QPSK with Rayleigh fading.

BER as a function of the power ratio for DLT, where the best 527 performance is provided for $\alpha$ in the range of 0.2 for QPSK and 528 0.4 for 8PSK. Fig. 10 shows the goodput with increasing SNR, 529 where again it can be observed that the DLT provides better 530 goodput than SMX at higher SNR values. As above, for both 531 scenarios characterized in the figure, we also include the cases 532 where the symbol modulation order used for SMX and RSM is 533 increased for some of the spatial streams in order to achieve the 534 same BE values of $\beta=19$ and $\beta=27$ with the proposed DLT, 535 for QPSK and 8PSK, respectively. Again, it can be seen that the 536 proposed DLT scheme obtains the maximum goodput at lower 537 SNR values.

Finally, Figs. 11 and 12 show the PE of the SMX, RSM 539 and DLT techniques. Fig. 11 shows the PE for increasing 540 transmit power, within the region of power values used in 541 the communication standards for $(10 \times 8)$ and $(8 \times 4)$ MIMO 542 systems. It is assumed here that the noise variance is $\sigma^{2}=1$ to 543 indirectly account for the path loss (and, hence, the useful signal 544 power loss) experienced in real transmission. It can be seen that 545 the proposed DLT scheme outperforms SMX and RSM in terms 546 


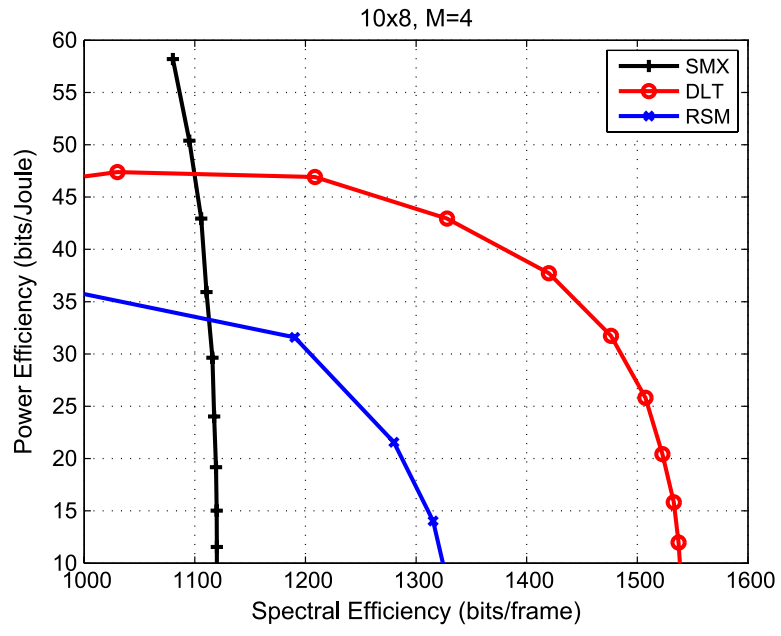

Fig. 12. Power efficiency versus spectral efficiency for a $(10 \times 8)$ MIMO with SMX and DLT, as well as QPSK with Rayleigh fading.

547 of $\mathrm{PE}$ for all transmit power values in both $(10 \times 8)$ and $(8 \times 4)$ 548 MIMO systems. The tradeoff between PE and BE is shown in 549 Fig. 12. It can be seen that DLT offers a more scalable tradeoff 550 with a wider range of $\mathrm{BEs}$ for the $\mathrm{PE}$ range, while it is more 551 power efficient than SMX and RSM in the region of high BEs.

\section{CONCLUSION}

553 A dual-layered DL transmission scheme was proposed, 554 which combines traditional MIMO SMX with RSM. As op555 posed to traditional SM where a subset of antennas carry a spa556 tial stream, here, we allow all antennas to carry information by 557 applying SM on the symbol power-level domain. This provides 558 scope for the analytical optimization of the ratio between the 559 power levels used in the proposed scheme. Both our simulations 560 and performance analysis show that, by allowing all antennas 561 to form spatial streams, the proposed scheme improves the 562 system's BE and power efficiency compared to both SMX 563 and SM.

564 Further work can involve exploring more advanced TPC 565 schemes for the proposed transmission scheme and exploring 566 the adaptations of the proposed scheme for QAM and enhanc567 ing its robustness to channel state information errors.

\section{REFERENCES}

[6] A. Garcia and C. Masouros, "Power-efficient Tomlinson-Harashima 584 precoding for the downlink of multi-user MISO systems," IEEE Trans. 585 Commun., vol. 62, no. 6, pp. 1884-1896, Jun. 2014.

[7] C. B. Peel, B. M. Hochwald, and A. L. Swindlehurst, "A vector- 587 perturbation technique for near-capacity multiantenna multiuser 588 communication-Part I: Channel inversion and regularization," IEEE 589 Trans. Commun., vol. 53, no. 1, pp. 195-202, Jan. 2005.

[8] C. Masouros and E. Alsusa, "Dynamic linear precoding for the exploita- 591 tion of known interference in MIMO broadcast systems," IEEE Trans. 592 Wireless Commun., vol 8, no. 3, pp. 1396-1404, Mar. 2009.

[9] C. Masouros and E. Alsusa, "Soft transmitter precoding for the down- 594 link of DS/CDMA communication systems," IEEE Trans. Veh. Technol., 595 vol. 59, no. 1, pp. 203-215, Jan. 2010.

[10] C. Masouros, T. Ratnarajah, M. Sellathurai, C. Papadias, and A. Shukla, 597 "Known interference in wireless communications: A limiting factor or a 598 potential source of green signal power?" IEEE Commun., Mag., vol. 51, 599 no. 10 , pp. $162-171$, Oct. 2013.

[11] G. Zheng et al., "Rethinking the role of interference in wireless networks," 601 IEEE Commun. Mag., vol. 52, no. 11, pp. 152,158, Nov. 2014.

[12] C. Masouros, "Correlation rotation linear precoding for MIMO broadcast 603 communications," IEEE Trans. Signal Process., vol 59, no. 1, pp. 252-262, 604 Jan. 2011.

[13] F. Rusek et al., "Scaling up MIMO: Opportunities and challenges with 606 very large arrays," IEEE Signal Process. Mag., vol. 30, no. 1, pp. 40-60, 607 Jan. 2013.

[14] C. Masouros, M. Sellathurai, and T. Ratnarajah, "Large-scale MIMO 609 transmitters in fixed physical spaces: The effect of transmit correlation and 610 mutual coupling," IEEE Trans. Commun., vol. 61, no. 7, pp. 2794-2804, 611 Jul. 2013.

[15] C. Masouros and M. Matthaiou, "Physically constrained massive MIMO: 613 Hitting the wall of favorable propagation," IEEE Commun. Let., vol. 19, 614 no. 5, pp. 771-774, May 2015.

[16] R. Mesleh, H. Haas, S. Sinanovic, C. W. Ahn, and S. Yun, "Spatial 616 modulation," IEEE Trans. Veh. Technol., vol. 57, no. 4, pp. 2228-2241, 617 Jul. 2008.

[17] M. Di Renzo, H. Haas, A. Ghrayeb, S. Sugiura, and L. Hanzo, "Spatial 619 modulation for generalized MIMO: Challenges, opportunities, and imple- 620 mentation," Proc. IEEE, vol. 102, no. 1, pp. 56-103, Jan. 2014.

621

[18] A. Garcia and C. Masouros, "Low-complexity compressive sensing detec- 622 tion for spatial modulation in large-scale multiple access channels," IEEE 623 Trans. Commun., to be published.

[19] M. Di Renzo and H. Haas, "Bit error probability of space modulation over 625 Nakagami-m fading: Asymptotic analysis," IEEE Commun. Lett., vol. 15, 626 no. 10 , pp. 1026-1028, Oct. 2011.

[20] J. Jeganathan, A. Ghrayeb, and L. Szczecinski, "Spatial modulation: 628 Optimal detection and performance analysis," IEEE Commun. Lett., 629 vol. 12, no. 8, pp. 545-547, Aug. 2008.

[21] A. Younis, S. Sinanovic, M. Di Renzo, R. Mesleh, and H. Haas, "Gen- 631 eralised sphere decoding for spatial modulation," IEEE Trans. Commun., 632 vol. 61, no. 7, pp. 2805-2815, Jul. 2013.

[22] J. Wang, S. Jia, and J. Song, "Generalised spatial modulation system 634 with multiple active transmit antennas and low complexity detection 635 scheme," IEEE Trans. Wireless Commun., vol. 11, no. 4, pp. 1605-1615, 636 Apr. 2012.

[23] M. Di Renzo and H. Haas, "On transmit diversity for spatial modulation 638 MIMO: Impact of spatial constellation diagram and shaping filters at the 639 transmitter," IEEE Trans. Veh. Technol., vol. 62, no. 6, pp. 2507-2531, 640 Jul. 2013.

641

[24] P. Yang et al., "Star-QAM signaling constellations for spatial mod- 642 ulation," IEEE Trans. Veh. Technol., vol. 63, no. 8, pp. 3741-3749, 643 Oct. 2014.

[25] S. Sugiura, C. Xu, S. X. Ng, and L. Hanzo, "Reduced-complexity coherent 645 versus non-coherent QAM-aided space-time shift keying," IEEE Trans. 646 Commun., vol. 59, no. 11, pp. 3090-3101, Nov. 2011.

[26] K. Ntontin, M. Di Renzo, A. Perez-Neira, and C. Verikoukis, "Adaptive 648 generalized space shift keying," EURASIP J. Wireless Commun. Netw., 649 vol. 2013, no. 1, p. 43, Feb. 2013.

[27] S. Sugiura and L. Hanzo, "On the joint optimization of dispersion 651 matrices and constellations for near-capacity irregular precoded space- 652 time shift keying," IEEE Trans. Wireless Commun., 2013, vol. 12, no. 1, 653 pp. 380-387, Jan. 2013.

[28] M. Maleki, H. Bahrami, S. Beygi, M. Kafashan, and N. H. Tran, "Space 655 modulation with CSI: Constellation design and performance evaluation," 656 IEEE Trans. Veh. Technol., vol. 62, no. 4, pp. 1623-1634, May 2013. 657

[29] X. Guan, Y. Cai, and W. Yang, "On the mutual information and precoding 658 for spatial modulation with finite alphabet," IEEE Wireless Commun. 659 Lett., vol. 2, no. 4, pp. 383-386, Aug. 2013. 
661 [30] J. M. Luna-Rivera, D. U. Campos-Delgado, and M. G. Gonzalez-Perez, 662 "Constellation design for spatial modulation," Procedia Technol., vol. 7, 663 pp. 71-78, 2013.

664 [31] C. Masouros, "Improving the diversity of spatial modulation in MISO 665 channels by phase alignment," IEEE Commun. Lett., vol. 18, no. 5, 666 pp. 729-732, May 2014.

667 [32] A. Garcia, C. Masouros, and L. Hanzo, "Pre-scaling optimization for space 668 shift keying based on semidefinite relaxation," IEEE Trans. Commun., 669 to be published.

670 [33] C. Masouros and L. Hanzo, "Constellation-randomization achieves 671 transmit diversity for single-RF spatial modulation," IEEE Trans. Veh. 672 Technol., to be published.

673 [34] J. Jeganathan, A. Ghrayeb, L. Szczecinski, and A. Ceron, "Space shift 674 keying modulation for MIMO channels," IEEE Trans. Wireless Commun., 675 vol. 8, no. 7, pp. 3692-3703, Jul. 2009.

676 [35] M. Di Renzo and H. Haas, "Bit error probability of space modulation over 677 Nakagami-m fading: Asymptotic analysis," IEEE Commun. Lett., vol. 15, 678 no. 10, pp. 1026-1028, Oct. 2011.

679 [36] R. Zhang, L. Yang, and L. Hanzo, "Generalised pre-coding aided spatial 680 modulation," IEEE Trans. Wireless Commun., vol. 12, no. 11, pp. 54346815443 , Nov. 2013.

682 [37] D.-T. Phan-Huy and M. Helard, "Receive antenna shift keying for 683 time reversal wireless communications," in Proc. IEEE ICC, Jun. 2012, $684 \quad$ pp. $4852-4856$.

685 [38] R. Zhang, L. L. Yang, and L. Hanzo, "Error probability and capacity 686 analysis of generalised pre-coding aided spatial modulation," IEEE Trans. 687 Wireless Commun., vol. 14, no. 1, pp. 364-375, Jan. 2015.

688 [39] A. Stavridis, S. Sinanovic, M. Di Renzo, and H. Haas, "Transmit precod689 ing for receive spatial modulation using imperfect channel knowledge," in 690 Proc. IEEE Veh. Technol. Conf. Spring, May 2012, pp. 1-5.

691 [40] R. M. Legnain, R. H. M. Hafez, I. D. Marsland, and A. M. Legnain, 692 "A novel spatial modulation using MIMO spatial multiplexing," in Proc. 693 ICCSPA, Feb. 2013, pp. 1-4.

694 [41] J. G. Proakis, Digital Communications, Electrical Engineering, 3rd ed. 695 New York, NY, USA: McGraw-Hill, 1995.

696 [42] M. Abramowitz and I. A. Stegun, Handbook of Mathematical Functions 697 With Formulas, Graphs, and Mathematical Tables. New York, NY, USA: 698 Dover, 1972.

699 [43] X. Cong, G. Y. Li, Z. Shunqing, Y. Chen, and S. Xu, "Energy- and 700 spectral-efficiency tradeoff in downlink OFDMA networks," IEEE Trans. 701 Wireless Commun., vol. 10, no. 11, pp. 3874-3886, Nov. 2011.

702 [44] S. Cui, A. J. Goldsmith, and A. Bahai, "Energy-constrained modulation 703 optimization," IEEE Trans. Wireless Commun., vol. 4, no. 5, pp. 23497042360, Sep. 2005.

705 [45] C. Masouros, M. Sellathurai, and T. Ratnarajah, "Computationally ef706 ficient vector perturbation precoding using thresholded optimization," 707 IEEE Trans. Commun., vol. 61, no. 5, pp. 1880-1890, May 2013.

708 [46] C. Masouros, M. Sellathurai, and T. Ratnarajah, "Vector perturbation 709 based on symbol scaling for limited feedback MIMO downlinks," IEEE 710 Trans. Signal Process., vol. 62, no. 3, pp. 562-571, Feb. 1, 2014.

711 [47] D. Curd, Power consumption in 65 nm FPGAs, Xilinx White Paper, 712 Feb. 2007

713 [48] Evolved Universal Terrestrial Radio Access (E-UTRA); LTE Physical

714 Layer; General Description, Third-Generation Partnership Project, TS $715 \quad 36.201$, V11.1.0 (2008-03), Rel. 11, 2008.

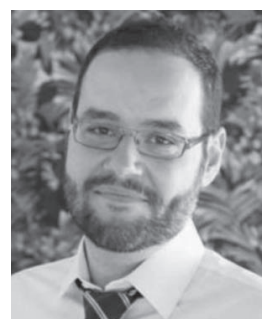

Christos Masouros (M'06-SM'14) received the 716 Diploma in electrical and computer engineering 717 from the University of Patras, Patras, Greece, in 718 2004 and the M.Sc. and Ph.D. degrees in electrical 719 and electronic engineering from The University of 720 Manchester, Manchester, U.K., in 2006 and 2009, 721 respectively.

722

He was a Research Associate with the University 723 of Manchester and a Research Fellow with Queen's 724 University Belfast, Belfast, U.K. He is currently a 725 Lecturer with the Department of Electrical and Elec- 726 tronic Engineering, University College London, London, U.K. His research in- 727 terests include wireless communications and signal processing, with particular 728 focus on green communications, large-scale antenna systems, cognitive radio, 729 and interference mitigation techniques for multiple-input-multiple-output and 730 multicarrier communications.

Dr. Masouros is the Principal Investigator of the EPSRC Project 732 EP/M014150/1 on large-scale antenna systems. He received a Royal Academy 733 of Engineering Research Fellowship for 2011-2016.

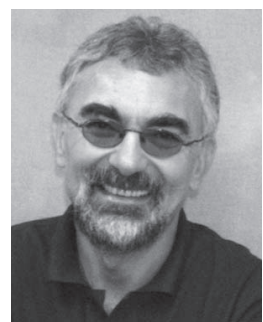

Lajos Hanzo (M'91-SM'92-F'04) received the 735 M.S. degree in electronics and the Ph.D. degree from 736 Budapest University of Technology and Econom- 737 ics (formerly, Technical University of Budapest), 738 Budapest, Hungary, in 1976 and 1983, respectively; 739 the D.Sc. degree from the University of Southampton, 740 Southampton, U.K., in 2004; and the "Doctor Honoris 741 Causa" degree from Budapest University of Technol- 742 ogy and Economics in 2009.

743

During his 38-year career in telecommunications, 744 he has held various research and academic posts in 745 Hungary, Germany, and the U.K. Since 1986, he has been with the School 746 of Electronics and Computer Science, University of Southampton, where he 747 holds the Chair in Telecommunications. He is currently directing a 100-strong 748 academic research team, working on a range of research projects in the field of 749 wireless multimedia communications sponsored by industry, the Engineering 750 and Physical Sciences Research Council of U.K., the European Research 751 Council's Advanced Fellow Grant, and the Royal Society Wolfson Research 752 Merit Award. During 2008-2012, he was a Chaired Professor with Tsinghua 753 University, Beijing, China. He is an enthusiastic supporter of industrial and 754 academic liaison and offers a range of industrial courses. He has successfully 755 supervised more than $80 \mathrm{Ph} . D$. students, coauthored 20 John Wiley/IEEE Press 756 books on mobile radio communications, totaling in excess of 10000 pages, and 757 published more than 1400 research entries on IEEEXplore. He has more than 758 20000 citations. His research is funded by the European Research Council's 759 Senior Research Fellow Grant.

Dr. Hanzo is a Fellow of the Royal Academy of Engineering, The Institution 761 of Engineering and Technology, and the European Association for Signal 762 Processing. He is also a Governor of the IEEE Vehicular Technology Society. 763 He has served as the Technical Program Committee Chair and the General Chair 764 of IEEE conferences, has presented keynote lectures, and has been awarded a 765 number of distinctions. During 2008-2012, he was the Editor-in-Chief of the 766 IEEE Press. 


\section{AUTHOR QUERIES}

\section{AUTHOR PLEASE ANSWER ALL QUERIES}

AQ1 = Please provide publication update in Ref. [18].

AQ2 = Please provide publication update in Ref. [32].

AQ3 = Please provide publication update in Ref. [33].

\section{END OF ALL QUERIES}




\title{
Dual-Layered MIMO Transmission for Increased Bandwidth Efficiency
}

\author{
Christos Masouros, Senior Member, IEEE, and Lajos Hanzo, Fellow, IEEE
}

\begin{abstract}
4 Abstract-A dual-layered downlink transmission scheme is 5 proposed for intrinsically amalgamating multiple-input-multiple6 output (MIMO) spatial multiplexing (SMX) with spatial modu7 lation (SM). The proposed scheme employs a classic SMX trans8 mission that is known to offer superior bandwidth efficiency (BE) 9 compared with SM. We exploit receive-antenna-based SM (RSM) 10 on top of this transmission as an enhancement of the BE. The RSM 11 here is applied to the combined spatial and power-level domain not 12 by activating and deactivating the RAs but rather by choosing be13 tween two power levels $\left\{\boldsymbol{P}_{\mathbf{1}}, \boldsymbol{P}_{\mathbf{2}}\right\}$ for the received symbols in these 14 antennas. In other words, the combination of symbols received at 15 a power level $\boldsymbol{P}_{\boldsymbol{1}}$ carries information in the spatial domain in the 16 same manner as the combination of nonzero elements in the re17 ceive symbol vector carries information in the RSM transmission. 18 This allows for the coexistence of RSM with SMX, and the results 19 show increased BE for the proposed scheme compared with both 20 SMX and SM. To characterize the proposed scheme, we carry out 21 a mathematical analysis of its performance, and we use this to 22 optimize the ratio between $\boldsymbol{P}_{1}$ and $\boldsymbol{P}_{2}$ for attaining the minimum 23 error rates. Our analytical and simulation results demonstrate 24 significant BE gains for the proposed scheme compared with 25 conventional SMX and SM.
\end{abstract}

26 Index Terms-Multiple-input-multiple-output systems, spatial 27 modulation (SM), spatial multiplexing (SMX), transmit precoding 28 (TPC).

\section{INTRODUCTION}

$301 \mathrm{~V}$ ULTIANTENNA-aided transceivers have been shown to improve the capacity of the wireless channel by 33 means of spatial multiplexing (SMX) [1]. For the multiuser 34 downlink (DL), transmit precoding (TPC) schemes have been 35 shown to improve both the attainable power efficiency (PE) and 36 the cost of mobile terminals by shifting the signal processing 37 complexity to the base stations (BSs). Numerous TPC solutions

Manuscript received November 25, 2014; revised February 16, 2015 and April 20, 2015; accepted May 22, 2015. The work of C. Masouros was supported in part by the Royal Academy of Engineering, U.K., and in part by the Engineering and Physical Sciences Research Council (EPSRC) under Project EP/M014150/1. The work of L. Hanzo was supported in part by the India-U.K. Advanced Technology Centre, by the EU Concerto Project, by the European Research Council under the Advanced Fellow Grant, and by the Royal Society under the Wolfson Research Merit Award. The review of this paper was coordinated by Dr. N.-D. Đào.

C. Masouros is with the Department of Electrical and Electronic Engineering, University College London, London WC1E 7JE, U.K. (e-mail: chris. masouros@ieee.org).

L. Hanzo is with the School of Electronics and Computer Science, University of Southampton, Southampton SO17 1BJ, U.K. (e-mail: 1h@ecs.soton.ac.uk).

Color versions of one or more of the figures in this paper are available online at http://ieeexplore.ieee.org.

Digital Object Identifier 10.1109/TVT.2015.2438776 exist, ranging from highly complex capacity achieving nonlin- 38 ear dirty paper coding techniques [2] and their low-complexity 39 suboptimal counterparts in the form of Tomlinson-Harashima 40 precoding [3]-[6] to linear TPC schemes based on channel 41 inversion [7]-[12] that offers the lowest complexity, albeit at an 42 inferior performance. The performance-complexity tradeoffs 43 between the above TPC have been thoroughly studied in the 44 literature. More recently, it has been shown that the family 45 of linear techniques exhibits a close-to-optimal performance 46 in the large-scale multiple-input-multiple-output (MIMO) 47 region [13]-[15]. Accordingly, we focus on the class of low- 48 complexity closed-form linear TPC [7]-[12] due to their favor- 49 able performance-complexity tradeoff and practical relevance. 50

More recently, spatial modulation (SM) has been conceived 51 for implicitly encoding information in the index of the spe- 52 cific antenna activated for the transmission of the modulated 53 symbols, offering a low-complexity design alternative [16]. Its 54 central benefits include the absence of interantenna interference 55 (IAI) and the fact that it only requires a subset (down to 56 one) of radio-frequency (RF) chains compared with SMX. 57 Accordingly, the interantenna synchronization is also relaxed. 58 Early work has focused on the design of receiver algorithms for 59 minimizing the bit error ratio (BER) of SM at low complexity 60 [16]-[21]. The work spans from matched filtering as a low- 61 complexity technique for detecting the antenna index used for 62 SM [16] to the maximum likelihood (ML) [20] with a signif- 63 icantly reduced complexity compared with classic SMX ML 64 detectors, including compressive sensing approaches [18] and 65 performance analyses [19]. Reduced-space sphere detection has 66 also been proposed for SM in [21] for further complexity reduc- 67 tion where a generalized SM transmission was also explored 68 [22]. In addition to receive processing, recent work has also 69 proposed constellation shaping for SM [23]-[33]. Specifically, 70 the work on this topic has focused on three main directions: 71 shaping and optimization of the spatial constellation, i.e., the le- 72 gitimate sets of activated transmit antennas (TAs) [23], modula- 73 tion constellation shaping [24]-[28] for the SM and space shift 74 keying transmission, where the constellation of the modulated 75 bits is optimized, and joint spatial and modulation constellation 76 shaping, in the form of optimizing the received constellation 77 [29]-[33].

Closely related work has focused on applying the concept of 79 SM to the receive antennas (RAs) of the communication link, 80 as opposed to the TAs as per the above approaches, forming 81 the RA-based spatial modulation (RSM) concept [36]-[39]. By 82 means of TPC, this technique targets a specific subset of RAs, 83 which receive information symbols, whereas the rest of the RAs 84 
85 receive only noise. This may be achieved by using zero-forcing 86 (ZF) TPC and transmitting a combination of information sym87 bols and zeros to the RAs, depending on the spatial symbols 88 to convey. As opposed to conventional SM where a subset of $89 \mathrm{RF}$ chains is deployed, here, all TAs and RAs are active and 90 therefore there are no RF chain reductions. Still, the computa91 tional complexity of the receivers is drastically reduced, where 92 simply the indexes of the targeted RAs have to be detected, 93 and the classic symbols received at the activated RAs are then 94 demodulated.

95 Inspired by the above RSM philosophy, here, we propose 96 a dual-layered transmission (DLT) scheme, which intrinsically 97 amalgamates a full spatial multiplexing (SMX) with SM. First, 98 we note that since, for RSM, all TAs and RAs are active, there 99 are no RF chain reductions, and this motivates the full SMX 100 approach. To accommodate the SMX, we apply an SM to the 101 combined spatial and receive-power domain, where instead of 102 sending a combination of information symbols and zero power 103 to the RAs, we apply two different power levels for distinguish104 ing between the "active" and "inactive" RAs. In this manner, the 105 spatial symbols are formed based on the power levels detected. 106 We demonstrate that this improves the bandwidth efficiency 107 (BE) with respect to SMX and SM. Against this state of the 108 art, we list the main contributions of this paper.

110

111

112

113

114

115

116

117

118

119

120

121

122

123

124

125

126

127

128

129 130 on a single-link scenario, the proposed technique can be readily 131 extended to a multiuser DL scenario, where the DLT and the 132 related RSM take place on a per-user basis, as facilitated by the 133 ZF-TPC employed at the BS.

134 Remark 2: The proposed scheme does not consist of a power 135 allocation scheme in the sense of allocating power according 136 to the quality-of-service $(\mathrm{QoS})$ requirements of the user. This 137 power allocation may be applied in addition to the proposed 138 scheme in the multiuser scenario, where different users with 139 different QoS requirements employ different sets of power 140 levels $\left\{P_{1}, P_{2}\right\}$ accordingly.

141 Remark 3: To facilitate the proposed power-level modula142 tion, this paper focuses on phase shift keying (PSK) in terms of the classical symbol modulation. Its adaptation to quadrature 143 amplitude modulation (QAM) is not trivial since the variability 144 of the power levels for the classically modulated symbols 145 would hinder the detection of the power levels of the spatially 146 modulated symbols. Nevertheless, even for PSK modulation, 147 our results illustrate a wide range of achievable BEs for the 148 proposed scheme and an improved performance compared with 149 classical SMX associated with both PSK and QAM for the 150 same BE.

The remainder of this paper is organized as follows. Section II 152 presents the MIMO system model and introduces the RSM 153 transmission philosophy. Section III details the proposed DLT 154 scheme, whereas in Section IV, we present our analytical study 155 of the performance attained and the analytical optimization 156 of the power ratio for the proposed scheme. Section V detail 157 the complexity calculation and the study of the attainable PE. 158 Finally, Section VI presents our numerical results, whereas our 159 conclusions are offered in Section VII.

\section{System Model And Receive-Antenna-Based SPATIAL MODULATION}

\section{A. System Model}

Consider a MIMO system, where the transmitter and receiver 164 are equipped with $N_{t}$ and $N_{r}$ antennas, respectively. For sim- 165 plicity, unless stated otherwise, in this paper, we assume that the 166 transmit power budget is limited as $P=1$. For the case of 167 the closed-form TPCs of [7]-[12], it is required that $N_{t} \geq N_{r} .168$ The given channel is modeled as follows:

$$
\mathbf{y}=\mathbf{H t}+\mathbf{w}
$$

where $\mathbf{y}$ is the vector of received symbols in all RAs, and $\mathbf{H}$ is 170 the MIMO channel vector with elements $h_{m, n}$ representing the 171 complex channel coefficient between the $n$th TA and the $m$ th 172 RA. Furthermore, $\mathbf{t}$ is the vector of precoded transmit symbols 173 that will be discussed in the following, and $\mathbf{w} \sim \mathcal{C N}\left(0, \sigma^{2} \mathbf{I}\right) 174$ is the additive white Gaussian noise (AWGN) component at 175 the receiver, with $\mathcal{C N}\left(\mu, \sigma^{2}\right)$ denoting the circularly symmet- 176 ric complex Gaussian distribution with a mean of $\mu$ and a 177 variance of $\sigma^{2}$.

\section{B. Receive-Antenna-Based Spatial Modulation}

The block diagram of RSM as proposed in [36] is shown 180 in Fig. 1(a). RSM targets a subset of the RAs by sending 181 information symbols to these RAs and zero power to the rest 182 of the RAs. While for RSM all RAs have to be on to detect 183 the arrival of information symbols, for coherence with the 184 SM literature, we shall refer to the antennas as "active" and 185 "inactive," depending on whether they do or do not receive 186 information symbols, respectively. The specific combination of 187 RAs that do receive symbols implicitly conveys the symbol 188 transmitted in the spatial domain. The above RA subset trans- 189 mission is achieved by forming a supersymbol vector in the 190 form $\mathbf{s}_{m}^{k}=\mathbf{e}_{k} b_{m}=\left[0, \ldots, b_{m_{1}}, \ldots, 0, \ldots, b_{m_{2}}, \ldots, 0\right]^{T}$ with 191 $N_{a}$ nonzero elements, where $\mathbf{e}_{k}$ is a diagonal matrix of size 192 


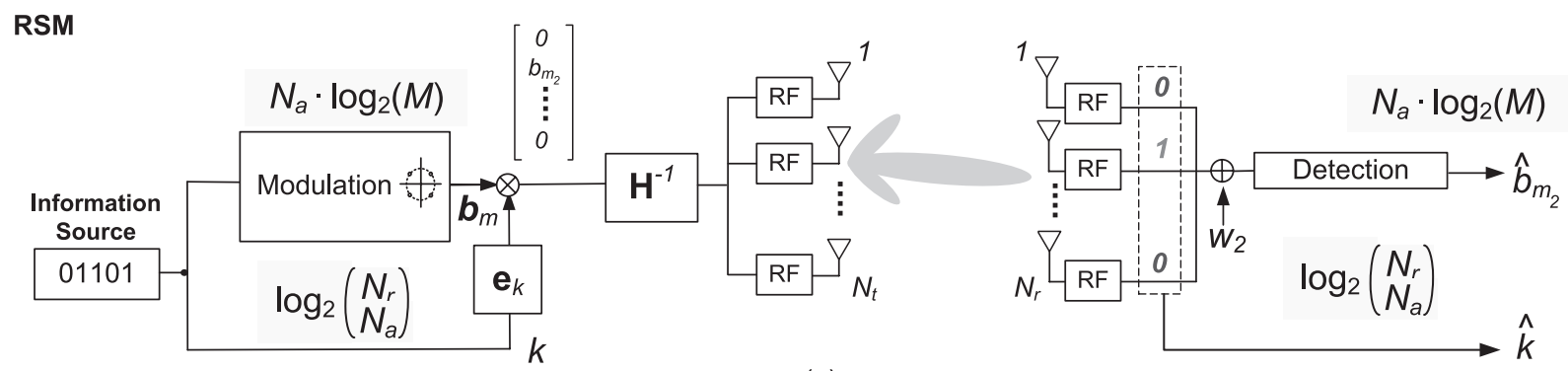

(a)

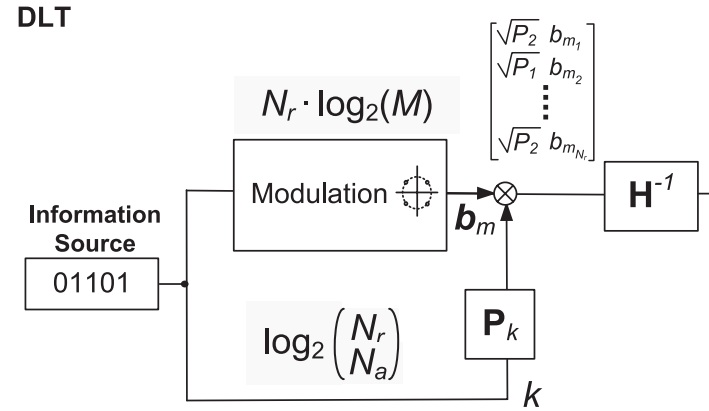

Fig. 1. Block diagram of (a) RSM and (b) DLT transmission.

$193 N_{r}$ with elements taken from the set $\{1,0\}$ on its diagonal, 194 which represents the RAs that are activated. The notation $[\cdot]^{T}$ 195 denotes the transpose operator. Here, $b_{m_{i}}, m_{i} \in\{1, \ldots, M\}$ 196 is a symbol taken from an $M$-order modulation alphabet that 197 represents the transmitted waveform in the baseband domain 198 conveying $\log _{2}(M)$ bits and $k$ represents the index of the $199 N_{a}$ activated RAs (the index of the nonzero elements in $\mathbf{s}_{m}^{k}$ ) 200 conveying $\log _{2}\left(\begin{array}{l}N_{r} \\ N_{a}\end{array}\right)$ bits in the spatial domain. Accordingly, 201 the total number of bits conveyed per supersymbol for RSM is

$$
\beta=N_{a} \log _{2}(M)+\log _{2}\left(\begin{array}{c}
N_{r} \\
N_{a}
\end{array}\right) .
$$

202 The transmitter then sends

$$
\mathbf{t}=f \mathbf{T} \mathbf{s}_{m}^{k}
$$

203 where $\mathbf{T}=\mathbf{H}^{H}\left(\mathbf{H H}^{H}\right)^{-1}$ is the ZF-TPC [7] that preserves 204 the form of $\mathbf{s}_{m}^{k}$ at the receiver. The factor $f=\sqrt{1 / \operatorname{tr}\left(\mathbf{T} \mathbf{T}^{H}\right)}$, 205 where $\operatorname{tr}(\cdot)$ denotes the trace operator and normalizes the 206 average transmit power to $P=1$. The received symbol vector 207 can be written as

$$
\mathbf{y}=f \mathbf{H T} \mathbf{s}_{m}^{k}+\mathbf{w}=f \mathbf{s}_{m}^{k}+\mathbf{w}
$$

208 where, clearly, all IAI is removed. At the receiver, a joint ML 209 detection of both the RA index and the transmit symbol is 210 obtained by the following minimization:

$$
\begin{aligned}
{\left[\hat{s}_{m}, \hat{k}\right] } & =\arg \min _{i}\left\|\mathbf{y}-\dot{\mathbf{y}}_{i}\right\| \\
& =\arg \min _{m_{i}, k_{i}}\left\|\mathbf{y}-f \mathbf{H T} \mathbf{s}_{m_{i}}^{k_{i}}\right\|
\end{aligned}
$$

211 where $\|\mathbf{x}\|$ denotes the norm of vector $\mathbf{x}$, and $\dot{\mathbf{y}}_{i}$ is the $i$ th 212 constellation point in the received SM constellation. A low- complexity decoupled approach is also proposed in [36], where 213 the first active antenna indexes are detected in the form of 214

$$
\hat{k}=\arg \max _{j \in \mathcal{J}} \sum_{i=1}^{N_{a}}\left|y_{j, i}\right|^{2}
$$

where $\mathcal{J}$ denotes the set of symbols in the spatial domain, and 215 then, the classic modulated symbols are detected by

$$
\hat{b}_{m_{i}}=\arg \min _{n_{i} \in \mathcal{Q}}\left|y_{\hat{k}, i} / f-b_{n_{i}}\right|^{2}
$$

where $\mathcal{Q}$ denotes the modulation constellation, and $b_{n_{i}}$ are 217 the symbols in the modulated symbol alphabet. For reasons of 218 computational complexity, we shall focus on the latter detector 219 in this paper.

\section{Proposed DuAL-LAYERED TRANSMission}

From the above system description, it can be seen that for 222 the particular case of RSM, while the detection complexity 223 is clearly reduced with respect to SMX, there are no savings 224 in RF complexity since all $N_{r}$ RAs have to be activated and 225 receiving for the detection in (6) and (7). Still, by forming a 226 subset of beams towards the receiver, as shown in Fig. 1(a), 227 the BE, i.e., the number of bits per channel use, is generally 228 lower for RSM than for SMX. Motivated by this, we propose 229 a dual-layered approach combining SMX with RSM, where 230 the BE of conventional SMX MIMO transmission is strictly 231 enhanced by encoding spatial bits in the RSM fashion in the 232 received power domain, by selecting two distinct, nonzero 233 power levels for the transmitted supersymbols instead of the 234 "on-off" RSM transmission in the $\{1,0\}$ manner. This allows 235 for nonzero elements throughout the supersymbol vector $\mathbf{s}_{m}^{k}, 236$ 
237 hence supporting a full SMX transmission in the modulated 238 signal domain. The block diagram of the proposed DLT is 239 shown in Fig. 1(b).

240 1) Transmitter: Here, we employ a full data vector in the 241 form of $\mathbf{b}_{m}=\left[b_{m_{1}}, b_{m_{2}}, \ldots, b_{m_{N_{r}}}\right]^{T}$, with all elements being 242 nonzero, and the encoding of the spatial bits is achieved by allo243 cating different power levels to the received symbols according 244 to the spatial symbol $k$, by applying the power allocation matrix $245 \mathbf{P}_{k}$, i.e.,

$$
\mathbf{s}_{m}^{k}=\mathbf{P}_{k} \mathbf{b}_{m}=\left[s_{m_{1}}, s_{m_{2}}, \ldots, s_{m_{N_{r}}}\right]^{T}
$$

246 with

$$
\mathbf{P}_{k}=\left[\begin{array}{cccc}
\sqrt{p_{1}} & 0 & \ldots & 0 \\
0 & \sqrt{p_{2}} & \cdots & 0 \\
\vdots & \vdots & \ddots & \vdots \\
0 & 0 & \ldots & \sqrt{p_{N_{r}}}
\end{array}\right]
$$

247 where $p_{i}, i \in\left[1, N_{r}\right]$ are taken from the set $\left\{P_{1}, P_{2}\right\}$ according 248 to the spatial symbol $k$. Note that classic QoS-based power al249 location can be applied in addition to this process by employing 250 an additional power allocation matrix on top of (9). The receiver 251 can then remove this additional matrix by simple inversion, in 252 order to detect the spatial symbol. For notational simplicity and 253 to keep the focus of the discussion on the proposed concept, we 254 neglect QoS-based power allocation.

255 2) Receiver: At the receiver side, the explicit knowledge of 256 the power levels $\left\{P_{1}, P_{2}\right\}$ is not required, as long as the detector 257 can distinguish between the two power levels. The received 258 signal of (4) can be decomposed as

$$
\begin{aligned}
& y_{p}=f \sqrt{P_{1}} b_{m_{p}}+w_{p}, p \in \mathcal{A} \\
& y_{q}=f \sqrt{P_{2}} b_{m_{q}}+w_{q}, q \in \mathcal{I}
\end{aligned}
$$

259 where $\mathcal{A}$ and $\mathcal{I}$ denote the sets of "active" and "inactive" 260 antennas, respectively. Hence, the receive processing is similar 261 to the conceived one for RSM, with the difference that the 262 classic modulated symbols of all RAs have to be detected, as 263 opposed to those of $N_{a}$ antennas only for RSM. Accordingly, 264 the receiver first detects the set of antennas with the highest 265 received power levels and then detects the classic modulated 266 symbols at all RAs according to

$$
\hat{k}=\arg \max _{j \in \mathcal{J}} \sum_{i=1}^{N_{a}}\left|y_{j, i}\right|^{2}
$$

267 where $\mathcal{J}$ denotes the set of symbols in the spatial domain, and

$$
\hat{\mathbf{b}}_{m}=\arg \min _{n \in \mathcal{Q}}\left|\mathbf{y} / f-\mathbf{b}_{n}\right|^{2}
$$

268 where $\mathcal{Q}$ denotes the classic modulation constellation, and $b_{n}$ 269 are the symbols in the modulated symbol alphabet.
TABLE I

BE IN BITS PER CHANNEL USE FOR SMX, RSM, AND DLT

\begin{tabular}{|l||r|}
\hline & Bandwidth Efficiency (BE) \\
\hline \hline SMX & $\beta=N_{r} \log _{2}(M)$ \\
\hline RSM & $\beta=N_{a} \log _{2}(M)+\log _{2}\left(\begin{array}{l}N_{r} \\
N_{a}\end{array}\right)$ \\
\hline DLT & $\beta=N_{r} \log _{2}(M)+\log _{2}\left(\begin{array}{l}N_{r} \\
N_{a}\end{array}\right)$ \\
\hline
\end{tabular}

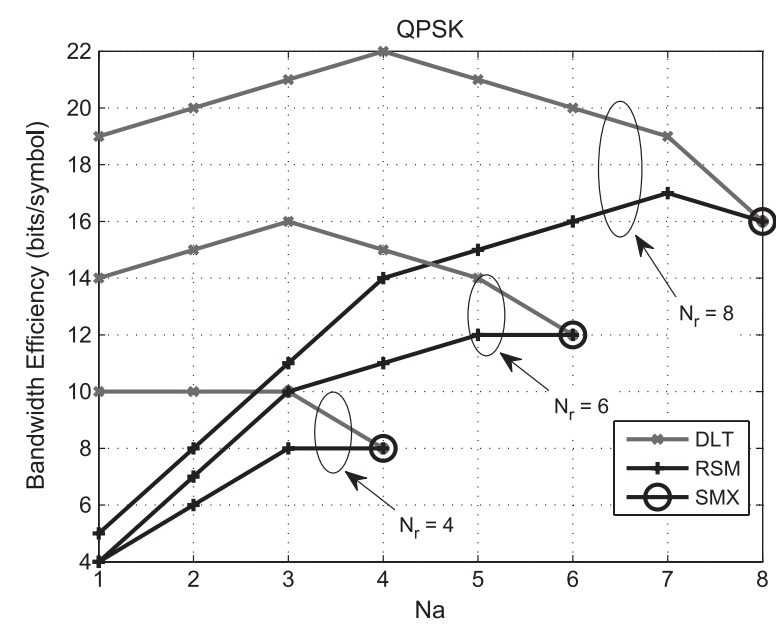

Fig. 2. BE versus $N_{a}$ for SMX, RSM, and DLT using the expressions of Table I.

\section{A. Bandwidth Efficiency}

Clearly, the encoding process in (8) and (9) encodes 271 $N_{r} \log _{2}(M)$ bits in the modulated symbol domain and an 272 additional $\log _{2}\left(\begin{array}{l}N_{r} \\ N_{a}\end{array}\right)$ bits in the spatial domain. This results in a 273 total of

$$
\beta=N_{r} \log _{2}(M)+\log _{2}\left(\begin{array}{c}
N_{r} \\
N_{a}
\end{array}\right)
$$

bits per transmitted supersymbol for DLT, which is strictly 275 greater than that for SMX and RSM. Here, the notation $N_{a} 276$ denotes the number of antennas receiving symbols at the power 277 level $P_{1}$. We should emphasize that, although all RAs are active 278 for both RSM and the proposed DLT, for coherence with the SM 279 literature, we shall adhere to the terms "active" and "inactive" 280 to indicate the antennas receiving $\{1,0\}$ and $\left\{P_{1}, P_{2}\right\}$ for RSM 281 and DLT, respectively. A comparison of the BEs of SMX, RSM, 282 and DLT is shown in Table I, where it can be seen that the 283 proposed DLT approach has an improved BE compared with 284 the conventional approaches. This is quantified in Fig. 2, where 285 the $\mathrm{BE}$ is expressed in terms of bits per channel use is shown 286 with increasing numbers of "active" antennas $N_{a}$ for MIMO 287 links with $N_{r}=4, N_{r}=6$, and $N_{r}=8$, where the clear bene- 288 fits of the proposed approach can be seen. It can be observed 289 that the additional BE of DLT compared with SMX can be 290 maximized by appropriately selecting the number of activated 291 antennas according to

$$
\tilde{N}_{a}=\arg \max _{N_{a}} \log _{2}\left(\begin{array}{c}
N_{r} \\
N_{a}
\end{array}\right)=N_{r} / 2
$$

which is demonstrated in the figure. 


\section{B. Symbol Power Levels}

295 With regard to the resulting BER performance, the set of 296 spatial power levels $\left\{P_{1}, P_{2}\right\}$ must be carefully selected so that 297 they satisfy a combination of two constraints.

299 1) There is sufficient separation between the two power lev300 els $P_{1}, P_{2}$ for correct detection of the "active" antennas $301 \quad$ and hence the spatial symbol $k$ in the presence of noise.

302 2) The symbols received with $P_{2}<P_{1}$ that dominate the 303 BER of the modulated symbol detection must experience 304 a sufficiently high signal-to-noise ratio (SNR) that is 305 adequate for reliable demodulation.

306 Let us therefore define the power ratio

$$
\alpha=\frac{P_{2}}{P_{1}}
$$

307 as the ratio between the two power levels transmitted, which 308 is optimized in the following results. Since $N_{a}$ symbols are 309 transmitted with power $P_{1}$ and the remaining $N_{r}-N_{a}$ symbols 310 have power of $P_{2}$, given a total power budget of $P=1$, we have

$$
\begin{aligned}
& P_{1}=\frac{1}{\left(N_{r}-N_{a}\right) \alpha+N_{a}} \\
& P_{2}=\frac{\alpha}{\left(N_{r}-N_{a}\right) \alpha+N_{a}} .
\end{aligned}
$$

311 Clearly, since the power levels $P_{1}, P_{2}$ influence the reliability 312 of detection for the modulated symbols and since the ratio $\alpha$ 313 determines the detection reliability of the spatial symbols, $\alpha$ 314 can be optimized for best BER performance. In the following, 315 we derive a closed-form expression for the optimum $\alpha$ value 316 for an $M$-order PSK modulation, where it can be seen that this 317 optimum value is independent of both $N_{r}$ and of $N_{a}$.

318 Remark: Regarding the effect of the above on the transmit 319 power distribution, we note that the power imbalance discussed 320 refers to the information symbols $\mathbf{s}_{m}^{k}$ and does not translate 321 to a power imbalance for the transmit symbols $t$. Indeed, the 322 ZF-precoded transmit symbols have the same average transmit 323 power, constrained by the scaling factor $f$ as shown above, 324 which is valid for both the proposed DLT and for the conven325 tional SMX, and these transmit symbols exhibit the same power 326 distribution for both techniques. In other words, the proposed 327 scheme does not impact the design of the power amplifiers used 328 at the transmitter.

329 To verify the above, Fig. 3 shows the probability density 330 function (pdf) of the normalized transmit power per antenna 331 for both SMX and DLT in a $(8 \times 4)$ element MIMO system. It 332 can be seen that, as expected, both techniques show the same 333 distribution of transmit power. \\ IV. DuAl-Layered Transmission PERFormanCE ANALYSIS AND OPTIMUM POWER RATIO $\alpha$}

\section{A. Probability of Error}

337 Here, we carry out a performance analysis for the proposed 338 DLT scheme by deriving the PEP between the pair of symbols

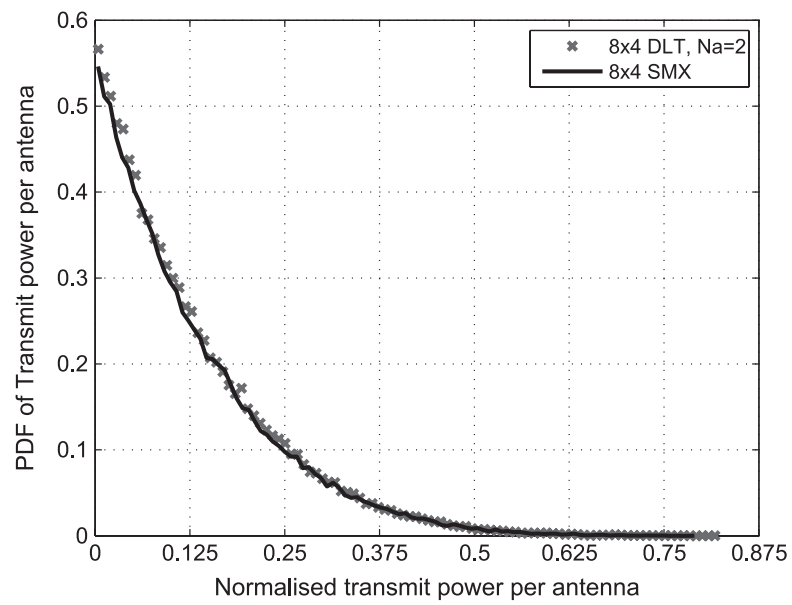

Fig. 3. PDF of transmit power per antenna for a $(8 \times 4)$ MIMO with SMX and DLT and QPSK with Rayleigh fading.

$\mathbf{s}_{m}^{k}$ and $\mathbf{s}_{n}^{l}$ in the superimposed spatial and classic modulation 339 constellations, following the analysis in [36]. Accordingly, we 340 define the PEP as $\mathcal{P}\left(\mathbf{s}_{m}^{k} \rightarrow \mathbf{s}_{n}^{l}\right)$ and use the union bound for the 341 average bit error probability $P_{e}$, which is expressed as

$$
P_{e} \leq \frac{1}{\beta} E\left\{\sum_{\mathbf{s}_{m}^{k} \in \mathcal{B}} \sum_{\mathbf{s}_{n}^{l} \in \mathcal{B} \neq \mathbf{s}_{m}^{k}} d\left(\mathbf{s}_{m}^{k}, \mathbf{s}_{n}^{l}\right) \mathcal{P}\left(\mathbf{s}_{m}^{k} \rightarrow \mathbf{s}_{n}^{l}\right)\right\}
$$

where $d\left(\mathbf{s}_{m}^{k}, \mathbf{s}_{n}^{l}\right)$ is the Hamming distance between the bit 343 representations of symbols $\mathbf{s}_{m}^{k}, \mathbf{s}_{n}^{l}$ and $\mathcal{B}=\mathcal{J} \cup \mathcal{Q}$ is the super- 344 symbol constellation defined as the union of the spatial domain 345 constellation and the classic modulation constellation. We have 346 used the operator $\cup$ to define the union of sets. For the PEP, we 347 have the following theorem.

348

Theorem 1: The PEP $\mathcal{P}\left(\mathbf{s}_{m}^{k} \rightarrow \mathbf{s}_{n}^{l}\right)$ for DLT can be expressed as 349

$\mathcal{P}\left(\mathbf{s}_{m}^{k} \rightarrow \mathbf{s}_{n}^{l}\right)=Q\left(\frac{f}{\sqrt{N_{0}}}\left(1-\sum_{i=1}^{N_{r}} \sqrt{p_{k_{i}} p_{l_{i}}} \mathcal{R}\left\{b_{m_{i}}^{*} b_{n_{i}}\right\}\right)\right)$

where $Q(\cdot)$ denotes the Gaussian $q$-function [42], $\mathcal{R}\{\cdot\}$ denotes 350 the real part of a number, $(\cdot)^{*}$ denotes the complex conjugate 351 operation, and $N_{0}=2 \sigma^{2}$ is the noise power spectral density. $\quad 352$

Proof: Let us first define $\mathbf{r}=\mathbf{y} / f$ and $\mathbf{v}=\mathbf{w} / f$ for 353 use in the following expressions. The PEP of the supersymbol 354 constellation can be expressed as

$$
\begin{aligned}
\mathcal{P}\left(\mathbf{s}_{m}^{k} \rightarrow \mathbf{s}_{n}^{l}\right)=\mathcal{P} & \left(\left\|\mathbf{r}-\mathbf{s}_{m}^{k}\right\|^{2}>\left\|\mathbf{r}-\mathbf{s}_{n}^{l}\right\|^{2}\right) \\
= & \mathcal{P}\left(\sum_{i=1}^{N_{r}} p_{k_{i}}\left|b_{m_{i}}\right|^{2}-2 \mathcal{R}\left\{r_{i}^{*} \sqrt{p_{k_{i}}} b_{m_{i}}\right\}\right. \\
& \left.>\sum_{i=1}^{N_{r}} p_{l_{i}}\left|b_{n_{i}}\right|^{2}-2 \mathcal{R}\left\{r_{i}^{*} \sqrt{p_{l_{i}}} b_{n_{i}}\right\}\right) .
\end{aligned}
$$

Since, for PSK signals, we have $\left|b_{m_{i}}\right|=1$, by rearranging 356 the terms in the probability expression, (21) can be further 357 
358 simplified as

$$
\begin{gathered}
\mathcal{P}\left(\mathbf{s}_{m}^{k} \rightarrow \mathbf{s}_{n}^{l}\right)=\mathcal{P}\left(\sum_{i=1}^{N_{r}} \mathcal{R}\left\{r_{i}^{*} \sqrt{p_{l_{i}}} b_{n_{i}}\right\}-\mathcal{R}\left\{r_{i}^{*} \sqrt{p_{k_{i}}} b_{m_{i}}\right\}\right. \\
\left.>\frac{\sum_{i=1}^{N_{r}} p_{l_{i}}-\sum_{i=1}^{N_{r}} p_{k_{i}}}{2}\right)
\end{gathered}
$$

359 Since $\sum_{i=1}^{N_{r}} p_{l_{i}}=\sum_{i=1}^{N_{r}} p_{k_{i}}=1$ and $r_{i}=\sqrt{p_{k_{i}}} b_{m_{i}}+v_{i}$, we 360 have

$$
\begin{gathered}
\mathcal{P}\left(\mathbf{s}_{m}^{k} \rightarrow \mathbf{s}_{n}^{l}\right)=\mathcal{P}\left(\sum_{i=1}^{N_{r}} \mathcal{R}\left\{\sqrt{p_{k_{i}}} b_{m_{i}}^{*} \sqrt{p_{l_{i}}} b_{n_{i}}\right\}+\mathcal{R}\left\{v_{i}^{*} \sqrt{p_{l_{i}}} b_{n_{i}}\right\}\right. \\
\left.>\sum_{i=1}^{N_{r}} p_{k_{i}}\left|b_{m_{i}}\right|^{2}+\mathcal{R}\left\{v_{i}^{*} \sqrt{p_{k_{i}}} b_{m_{i}}\right\}\right) \\
=\mathcal{P}\left(\sum_{i=1}^{N_{r}} \mathcal{R}\left\{v_{i}^{*}\left(\sqrt{p_{l_{i}}} b_{n_{i}}-\sqrt{p_{k_{i}}} b_{m_{i}}\right)\right\}\right. \\
\left.>1-\sum_{i=1}^{N_{r}} \sqrt{p_{k_{i}} p_{l_{i}}} \mathcal{R}\left\{b_{m_{i}}^{*} b_{n_{i}}\right\}\right) .
\end{gathered}
$$

361 Let us define the random variable $\chi \hat{=} \sum_{i=1}^{N_{r}} \mathcal{R}\left\{v_{i}^{*}\left(\sqrt{p_{l_{i}}} b_{n_{i}}-\right.\right.$ $\left.\left.362 \sqrt{p_{k_{i}}} b_{m_{i}}\right)\right\}$ for which we have $\chi \in \mathcal{N}\left(0, A N_{0} / f^{2}\right)$ with

$$
A=\frac{\sum_{i=1}^{N_{r}} p_{l_{i}}\left|b_{n_{i}}\right|^{2}+p_{k_{i}}\left|b_{n_{i}}\right|^{2}}{2}=\frac{1}{2} \sum_{i=1}^{N_{r}} p_{l_{i}}+p_{k_{i}} .
$$

363 For the unity transmit power assumed in this paper, it can be 364 seen from (24) that $A=1$. Accordingly, for the PEP, we have

$$
\mathcal{P}\left(\mathbf{s}_{m}^{k} \rightarrow \mathbf{s}_{n}^{l}\right)=\mathcal{P}\left(\chi>1-\sum_{i=1}^{N_{r}} \sqrt{p_{k_{i}} p_{l_{i}}} \mathcal{R}\left\{b_{m_{i}}^{*} b_{n_{i}}\right\}\right)
$$

365 which, for $\chi \in \mathcal{N}\left(0, N_{0} / f^{2}\right)$, leads to (20).

\section{B. Optimum Power Ratio $\alpha$}

367 As mentioned earlier, the power ratio $\alpha$ determines the 368 reliability of detection for the spatial symbol, whereas the lower 369 power level $P_{2}$ dominates the BER performance of the classic 370 modulated symbols' detection. As the probability of error in 371 (19) is dominated by the maximum PEP, the optimum power 372 ratio should be selected as

$$
\alpha_{\text {opt }}=\arg \min _{\alpha} \max _{\mathbf{s}_{m}^{k}, \mathbf{s}_{n}^{l}}\left\{\mathcal{P}\left(\mathbf{s}_{m}^{k} \rightarrow \mathbf{s}_{n}^{l}\right)\right\}
$$

373 To simplify the analysis, we shall treat the errors in the spatial 374 and classic modulated symbols separately. Accordingly, for the 375 maximum PEP $\mathcal{P}_{m}\left(s_{m_{i}}^{k} \rightarrow s_{m_{i}}^{l}\right)$ in the spatial domain only, we 376 have the following theorem.
Theorem 2: The maximum PEP $\mathcal{P}_{m}\left(s_{m_{i}}^{k} \rightarrow s_{m_{i}}^{l}\right)$ for the 377 spatial symbols in DLT can be expressed as

$$
\mathcal{P}_{m}\left(s_{m_{i}}^{k} \rightarrow s_{m_{i}}^{l}\right)=Q\left(\frac{f}{\sqrt{N_{0}}} \cdot \frac{\sqrt{P_{2}}-\sqrt{P_{1}}}{2}\right) .
$$

Proof: The maximum PEP in the spatial domain involves 379 the adjacent symbols of different power levels in the supersym- 380 bol constellation and can be expressed as

$$
\begin{aligned}
\mathcal{P}_{m} & \left(s_{m_{i}}^{k} \rightarrow s_{m_{i}}^{l}\right) \\
& =\mathcal{P}\left(\left\|r_{i}-s_{m_{i}}^{k}\right\|^{2}>\left\|r_{i}-s_{m_{i}}^{l}\right\|^{2}\right) \\
& =\mathcal{P}\left(P_{1}-2 \mathcal{R}\left\{r_{i}^{*} \sqrt{P_{1}} b_{m_{i}}\right\}>P_{2}-2 \mathcal{R}\left\{r_{i}^{*} \sqrt{P_{2}} b_{m_{i}}\right\}\right)
\end{aligned}
$$

where, using $r_{i}=\sqrt{p_{k_{i}}} b_{m_{i}}+v_{i}$, we get

$$
\begin{aligned}
\mathcal{P}_{m}\left(s_{m_{i}}^{k} \rightarrow s_{m_{i}}^{l}\right) & \\
= & \mathcal{P}\left(P_{1}-2 P_{1}\left|b_{m_{i}}\right|^{2}-2 \mathcal{R}\left\{u_{i}^{*} \sqrt{P_{1}} b_{m_{i}}\right\}\right. \\
& \left.>P_{2}-2 \sqrt{P_{1} P_{2}}\left|b_{m_{i}}\right|^{2}-2 \mathcal{R}\left\{u_{i}^{*} \sqrt{P_{2}} b_{m_{i}}\right\}\right) \\
= & \mathcal{P}\left(2\left(\sqrt{P_{2}}-\sqrt{P_{1}}\right) \mathcal{R}\left\{u_{i}^{*} b_{m_{i}}\right\}>P_{1}+P_{2}-2 \sqrt{P_{1} P_{2}}\right) \\
= & \mathcal{P}\left(-\mathcal{R}\left\{u_{i}^{*} b_{m_{i}}\right\}>\frac{\sqrt{P_{1}}-\sqrt{P_{2}}}{2}\right) .
\end{aligned}
$$

Similarly to the given proof, we have used the fact 383 that $\left|b_{m_{i}}\right|^{2}=1$, and it can be seen that $\psi \hat{=}-\mathcal{R}\left\{u_{i}^{*} b_{m_{i}}\right\} \in 384$ $\mathcal{N}\left(0, N_{0} / f^{2}\right)$. Accordingly, for the minimum PEP in the spatial 385 constellation, we have

$$
\mathcal{P}_{m}\left(s_{m_{i}}^{k} \rightarrow s_{m_{i}}^{l}\right)=\mathcal{P}\left(\psi>\frac{\sqrt{P_{2}}-\sqrt{P_{1}}}{2}\right)
$$

which leads to (27).

This indicates that the separation between $\left\{P_{1}, P_{2}\right\}$ should 388 be maximized for minimizing the errors in the spatial bits, 389 which are dominated by the distance between the pairs of adja- 390 cent symbols having different power levels $d_{s}=\sqrt{P_{1}}-\sqrt{P_{2}} .391$ We therefore define the spatial function $f_{S}(\alpha)$ that accounts for 392 the dependence of the spatial errors on $\alpha$ as

$$
f_{S}(\alpha) \triangleq \sqrt{P_{1}}-\sqrt{P_{2}}=\frac{1-\sqrt{\alpha}}{\sqrt{\left(N_{r}-N_{a}\right) \alpha+N_{a}}} .
$$

As regards to the classic modulated symbol errors, it is 394 known that the PSK error probability is given as [41]

$$
\begin{aligned}
\mathcal{P}\left(s_{m_{i}}^{k} \rightarrow s_{n_{i}}^{k}\right) & =\mathcal{P}\left(\left\|r_{i}-s_{m_{i}}^{k}\right\|^{2}>\left\|r_{i}-s_{n_{i}}^{l}\right\|^{2}\right) \\
& =Q\left(f \sqrt{\frac{P_{2}}{N_{0}} \log _{2}(M) \sin \frac{\pi}{M}}\right) .
\end{aligned}
$$




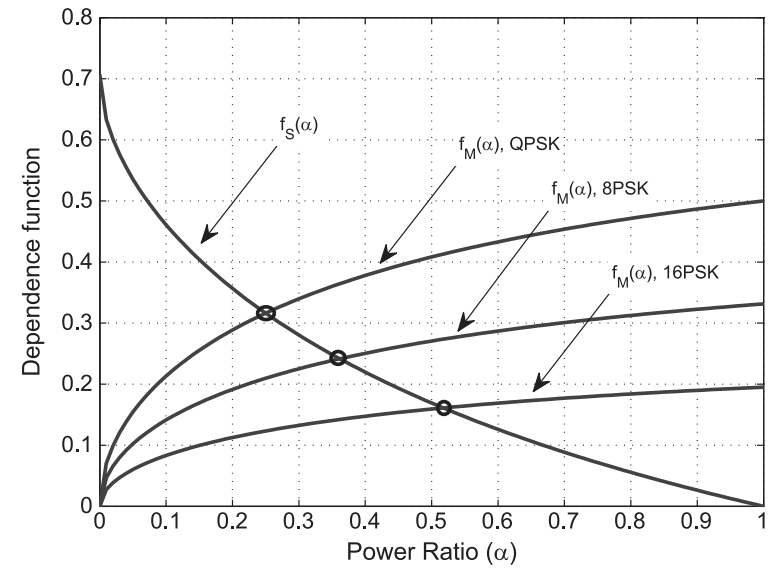

Fig. 4. Theoretical optimization of $\alpha$ for DLT for a $(8 \times 4)$ MIMO with $N_{a}=2$, using (36).

396 Accordingly, we define the function $f_{M}(\alpha)$ for the dependence 397 of the modulated symbol error on $\alpha$ as

$$
\begin{aligned}
f_{M}(\alpha) & \triangleq \sqrt{P_{2} \log _{2}(M) \sin \frac{\pi}{M}} \\
& =\sqrt{\log _{2}(M) \sin \frac{\pi}{M} \cdot \frac{\alpha}{\left(N_{r}-N_{a}\right) \alpha+N_{a}}} .
\end{aligned}
$$

398 The optimization (26) is equivalent to the maximization of 399 the minimum of these functions:

$$
\alpha_{\mathrm{opt}}=\arg \max _{\alpha}\left\{\min \left\{f_{S}(\alpha), f_{M}(\alpha)\right\}\right\} .
$$

400 The optimum power scaling ratio is, therefore, given as

$$
\begin{array}{r}
\alpha_{\mathrm{opt}}=\arg \max _{\alpha}\left\{\frac{1-\sqrt{\alpha}}{\sqrt{\left(N_{r}-N_{a}\right) \alpha+N_{a}}},\right. \\
\left.\sqrt{\log _{2}(M) \sin \frac{\pi}{M} \cdot \frac{\alpha}{\left(N_{r}-N_{a}\right) \alpha+N_{a}}}\right\}
\end{array}
$$

401 which is equivalent to selecting the factor $\alpha$ so that the two 402 terms in the minimization become equal, which gives

$$
\alpha_{\mathrm{opt}}=\frac{1}{\left(1+\sqrt{\log _{2}(M) \sin \frac{\pi}{M}}\right)^{2}} .
$$

403 We examine this optimization in Fig. 4, which shows the 404 functions $f_{s}(\alpha), f_{M}(\alpha)$ when increasing the values of $\alpha$ for 405 the example of a $(8 \times 4)$-element DLT system with $N_{a}=2$, 406 for $M=4,8,16$, i.e., quadrature phase-shift keying (QPSK), 407 8PSK, and 16PSK modulation. The intersections of the lines 408 determine the optimum values of $\alpha$. It will be shown in the 409 following that the theoretically obtained optimal values of $\alpha$ 410 closely match the optimal values obtained by simulation.

\section{COMPleXity AND POWER EFFICIENCY}

\section{A. Complexity}

413 Here, we compare the computational complexity of SMX, 414 RSM, and DLT and use this to carry out a comparison of the 415 resulting PE of the techniques. First, Table II summarizes the
TABLE II

COMPLEXITY FOR SMX, RSM, AND THE PROPOSED DLT SCHEME. $N_{\chi}=N_{a}$ FOR RSM, $N_{\chi}=N_{r}$ FOR DLT

\begin{tabular}{|l|r|}
\hline & Operations \\
\hline \hline Transmitter: & $N_{r}^{3}+2 N_{t} N_{r}$ \\
\hline ZF processing & $2 N_{a}\left(\begin{array}{l}N_{r} \\
N_{a}\end{array}\right)$ \\
\hline Receiver: & $N_{\chi} M$ \\
\hline Spatial detection & $C_{S M X}=N_{r}^{3}+N_{r}\left(2 N_{t}+M\right)$ \\
\hline Demodulation & $N_{r}\left(2\left(\begin{array}{c}N_{r} \\
N_{a}\end{array}\right)+M\right)$ \\
\hline \hline SMX Total & $\left.C_{R S M}=N_{r}^{3}+2 N_{t} N_{r}+N^{2}\right)$ \\
\hline RSM Total & $C_{D L T}=N_{r}^{3}+N_{r}\left(2 N_{t}+M\right)+2 N_{a}\left(\begin{array}{l}N_{r} \\
N_{a}\end{array}\right)$ \\
\hline DLT Total
\end{tabular}

computational complexity of each of the techniques, taking into 416 account the dominant operations at the transmitter and receiver. 417 We follow the typical assumption that multiplications and ad- 418 ditions require an equal number of floating point operations. 419 For all three schemes, the ZF-TPC employed at the transmitter 420 involves the inversion of the channel matrix that requires $N_{r}^{3}+421$ $N_{t} N_{r}$ operations and the multiplication with the supersymbol 422 vector involving an additional $N_{t} N_{r}$ operations. At the receiver, 423 all techniques require a demodulation stage that involves $M 424$ comparisons for and $M$-order modulation, for each antenna 425 receiving information, i.e., $N_{r} M$ for both SMX and DLT, and 426 $N_{a} M$ for RSM. The RSM and DLT require an additional stage 427 for the detection of the spatial symbol which, from (6) involves 428 $N_{a}$ complex multiplications and $N_{a}$ complex additions for each 429 antenna combination out of the $\left(\begin{array}{l}N_{r} \\ N_{a}\end{array}\right)$ combinations in total. 430

\section{B. Power Efficiency}

As the ultimate metric for evaluating the performance- 432 complexity tradeoff and the overall usefulness of the proposed 433 technique, we consider the PE of DLT compared with SMX and 434 RSM. Following the modeling of [43]-[46], we define the PE of 435 the communication link as the bit rate per total transmit power 436 dissipated, i.e., the ratio of the goodput achieved over the power 437 consumed:

$$
\mathcal{E}=\frac{T}{P_{\mathrm{PA}}+N_{t} \cdot P_{t}^{\mathrm{RF}}+N_{r} \cdot P_{r}^{\mathrm{RF}}+p_{c} \cdot C}
$$

where $P_{\mathrm{PA}}=((\xi / \eta)-1) P$ in Watts is the power dissipated by 439 the power amplifier to produce the total transmit signal power 440 $P$, with $\eta$ being the power amplifier's efficiency and $\xi$ being the 441 modulation-dependent peak-to-average power ratio (PAPR). 442 Furthermore, $P_{t}^{\mathrm{RF}}=P_{\text {mix }}+P_{\text {filt }}+P_{\mathrm{DAC}}$ and $P_{r}^{\mathrm{RF}}=P_{\text {mix }}+443$ $P_{\text {filt }}+P_{\mathrm{ADC}}$ represent the RF powers related to the mix- 444 ers, to the transmit filters, to the digital-to-analog con- 445 verter (DAC) at the transmitter and to the analog-to-digital 446 converter (ADC) at the receiver, which are assumed to 447 be constant for the purposes of this paper. We use prac- 448 tical values of these from [44] as $\eta=0.35$ and $P_{\text {mix }}=449$ $30.3 \mathrm{~mW}, P_{\text {filt }}=2.5 \mathrm{~mW}, P_{\mathrm{DAC}}=1.6 \mathrm{~mW}$, and $P_{\mathrm{DAC}}=450$ $1.3 \mathrm{~mW}$, yielding $P_{t}^{\mathrm{RF}}=34.4 \mathrm{~mW}$, and $P_{r}^{\mathrm{RF}}=34.1 \mathrm{~mW} .451$ In (37), $p_{c}$ in Watts/KOps is the power per $10^{3}$ elementary 452 
453 operations (KOps) of the digital signal processor, and $C$ is the 454 number of operations involved, taken from Table II, where 455 it is assumed that the operations shown dominate the digital 456 signal processing complexity of the link. This term is used 457 for introducing the complexity as a factor related to the power 458 dissipation in the PE metric. Typical values of $p_{c}$ include $p_{c}=$ $45922.88 \mathrm{~mW} / \mathrm{KOps}$ for the Virtex-4 and $p_{c}=5.76 \mathrm{~mW} / \mathrm{KOps}$ for 460 the Virtex-5 FPGA family from Xilinx [47]. Finally

$$
T=\beta B\left(1-P_{B}\right)=\beta B\left(1-P_{e}\right)^{B}
$$

461 represents the achieved goodput, where $P_{B}$ is the block error 462 rate with a block of size $B$ symbols, and $\beta$ is the BE of SM 463 in bits per symbol, taken from Table I. For reference, we have 464 assumed an LTE Type-2 TDD frame structure [48]. This has 465 a 10 ms duration that consists of 10 subframes, out of which 466 five subframes, containing 14 symbol time slots each, are used 467 for DL transmission yielding a block size of $B=70$ for the $468 \mathrm{DL}$, whereas the remainder are used for both uplink (UL) and 469 control information transmission. A slow fading channel is 470 assumed where the channel remains constant for the duration 471 of the frame.

472 The expression in (37) provides an amalgamated metric that 473 combines goodput, complexity, and transmit signal power, all 474 in a unified metric. High values of $\mathcal{E}$ indicate that high bit 475 rates are achievable for a given power consumption and thus 476 denote high energy efficiency. The following results show that 477 DLT provides an increased energy efficiency compared with 478 SMX and RSM in numerous scenarios using different transmit 479 power levels $P$.

\section{0 \\ VI. Numerical Results}

481 To evaluate the benefits of the proposed technique, this 482 section presents numerical results based on Monte Carlo sim483 ulations of SMX, RSM, and the proposed DLT. The channel 484 impulse response is assumed perfectly known at the transmitter. 485 Without loss of generality, unless stated otherwise, we assume 486 that the transmit power is restricted to $P=1$. MIMO systems 487 with up to eight TAs employing QPSK and 8PSK modulation 488 are explored, albeit it is plausible that the benefits of the 489 proposed technique extend to larger scale systems and higher 490 order modulation.

491 Remark: It should be noted that the BE improvement shown 492 in the following could also be obtained by SMX with the aid 493 of an increased classical modulation order. Accordingly, in the 494 following, we compare the proposed DLT to: (a) SMX using the 495 same classical modulation order to illustrate the improved BE 496 of DLT; and (b) SMX relying on a higher modulation order to 497 highlight the improved performance of DLT for an identical BE. 498 In Fig. 5, we show the BER as a function of the power 499 ratio for DLT for the $(8 \times 4)$ MIMO system, where the values 500 of $\alpha$ in the area of 0.25 can be seen to provide the best 501 performance. This matches well with the theoretically derived 502 result of Section IV-A and Fig. 4. Similarly, Fig. 6 shows the 503 BER versus $\alpha$ performance for higher order modulation 8PSK 504 and 16PSK. Again, a close match can be seen with the theo505 retically derived values for $\alpha_{\text {opt }}$. In Fig. 7, we show the BER 506 with increasing SNR for the proposed DLT, where the black

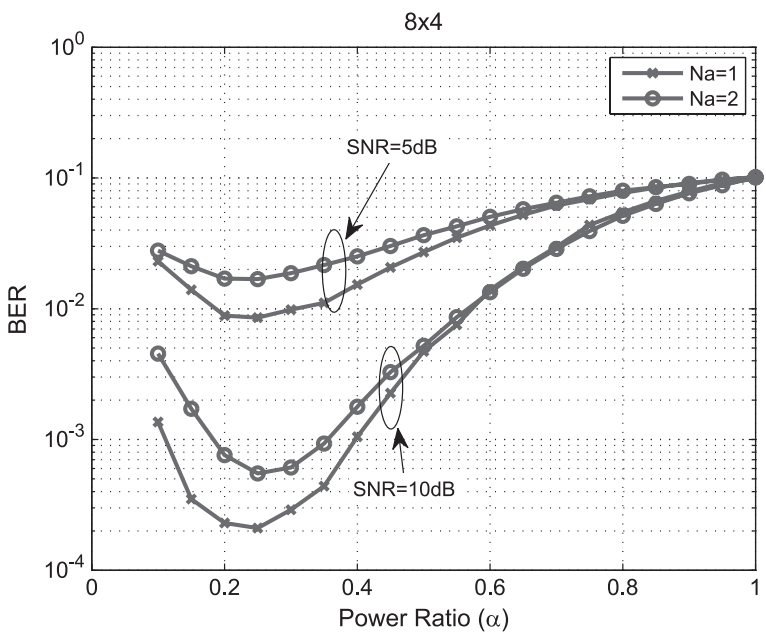

Fig. 5. BER versus $\alpha$ for an $(8 \times 4)$ MIMO with SMX and DLT, as well as QPSK with Rayleigh fading.
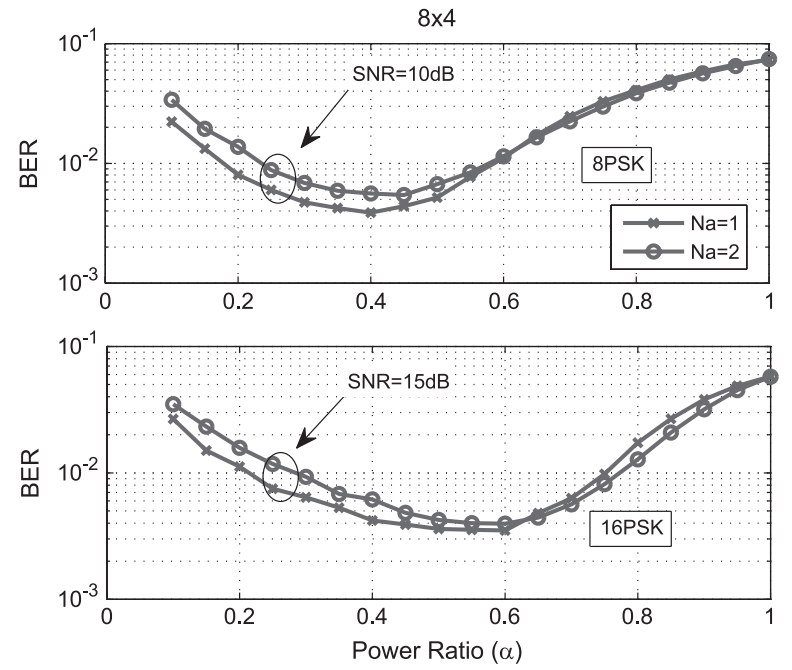

Fig. 6. BER versus $\alpha$ for a $(8 \times 4)$ MIMO with DLT, as well as 8 PSK and 16PSK with Rayleigh fading.

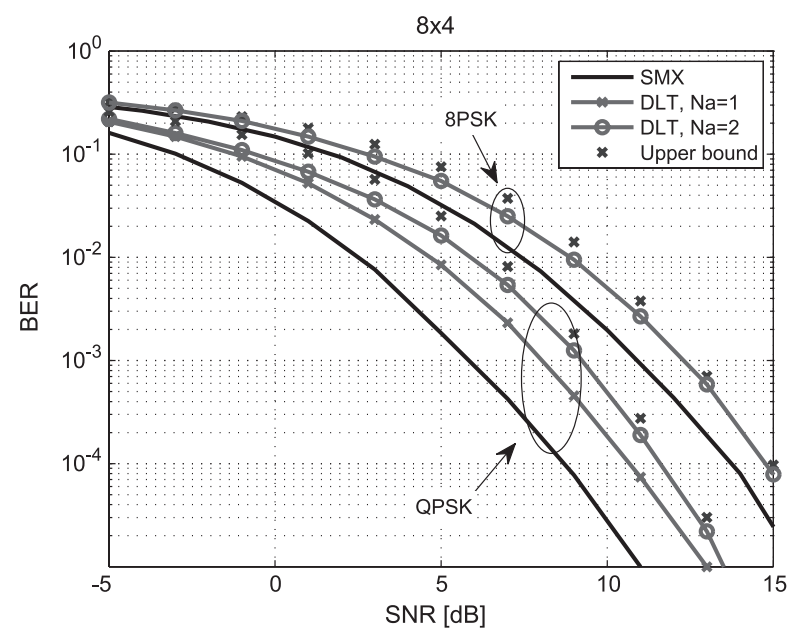

Fig. 7. BER versus SNR for a $(8 \times 4)$ MIMO with SMX and DLT, as well as QPSK and 8PSK with Rayleigh fading. 


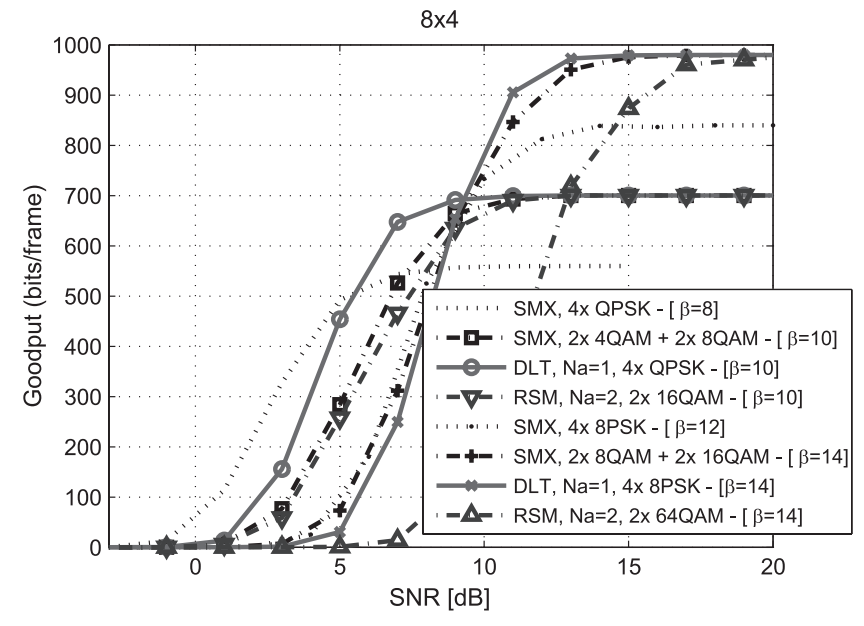

Fig. 8. Goodput versus SNR for a $(8 \times 4)$ MIMO with SMX and DLT, as well as Rayleigh fading.

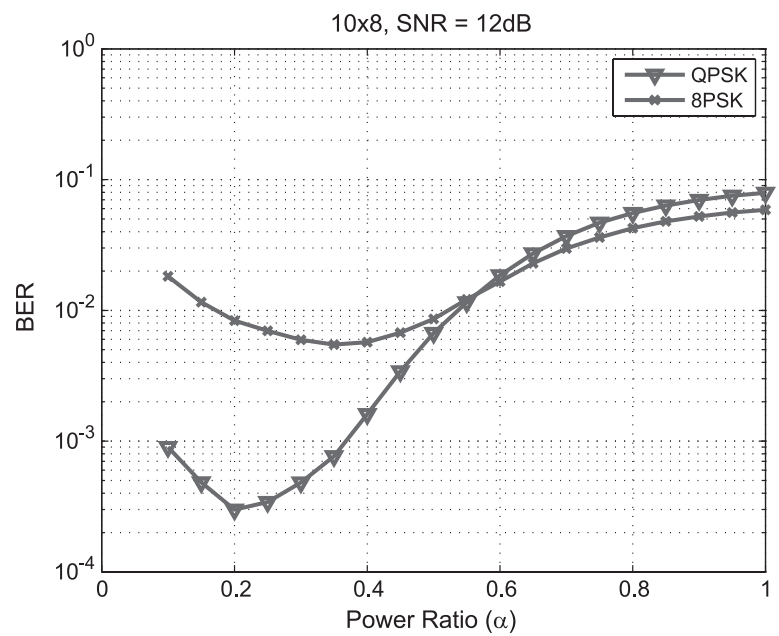

Fig. 9. BER versus $\alpha$ for a $(10 \times 8)$ MIMO with DLT, QPSK, and 8PSK with Rayleigh fading.

507 lines for $N_{a}=4$ represent SMX transmission. The curves show 508 results for both QPSK and 8PSK. The theoretical upper bound 509 using (19) is also depicted for both cases, and it can be observed 510 that it offers a tight bound. Clearly, the DLT scheme has inferior 511 BER performance compared with SMX due to the additional 512 spatial streams but at the benefit of improved BE. The improved $513 \mathrm{BE}$ of DLT is demonstrated in Fig. 8 where the goodput 514 with increasing SNR is depicted for the same $(8 \times 4)$ MIMO 515 scenario. Clearly, DLT provides higher goodput than SMX for 516 sufficiently high SNR values. To complete our comparisons, for 517 both scenarios in the figure, we also show the cases where the 518 symbol modulation order used for SMX and RSM is increased 519 for some of the spatial streams in order to achieve the same $520 \mathrm{BE}$ values of $\beta=10$ and $\beta=14$ with the proposed DLT, for 521 QPSK and 8PSK, respectively. Clearly, this has an impact on 522 the SNR requirement of SMX, where it can be seen that the 523 proposed DLT scheme obtains the maximum goodput at lower 524 SNR values.

525 The performance comparison is extended to the $(10 \times 8)$ 526 MIMO system in Figs. 9 and 10. In Fig. 9, we show the

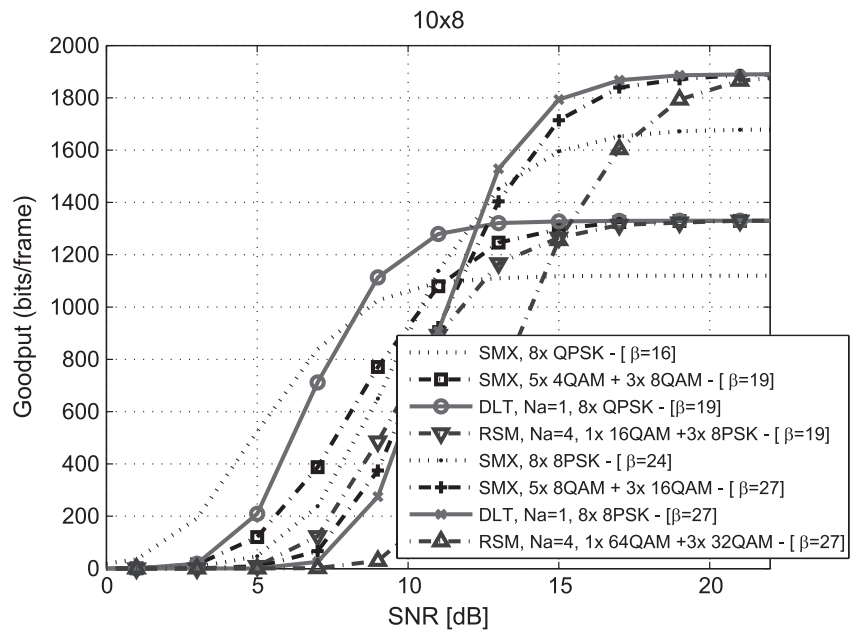

Fig. 10. Goodput versus SNR for a $(10 \times 8)$ MIMO with SMX and DLT, as well as Rayleigh fading.

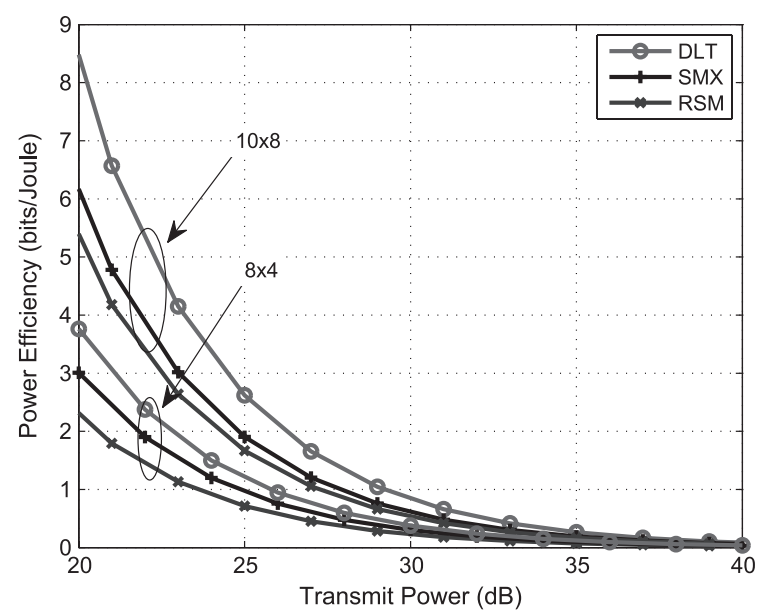

Fig. 11. PE versus transmit power for $(10 \times 8)$ and $(8 \times 4)$ MIMO systems with SMX and DLT, as well as QPSK with Rayleigh fading.

BER as a function of the power ratio for DLT, where the best 527 performance is provided for $\alpha$ in the range of 0.2 for QPSK and 528 0.4 for 8PSK. Fig. 10 shows the goodput with increasing SNR, 529 where again it can be observed that the DLT provides better 530 goodput than SMX at higher SNR values. As above, for both 531 scenarios characterized in the figure, we also include the cases 532 where the symbol modulation order used for SMX and RSM is 533 increased for some of the spatial streams in order to achieve the 534 same BE values of $\beta=19$ and $\beta=27$ with the proposed DLT, 535 for QPSK and 8PSK, respectively. Again, it can be seen that the 536 proposed DLT scheme obtains the maximum goodput at lower 537 SNR values.

Finally, Figs. 11 and 12 show the PE of the SMX, RSM 539 and DLT techniques. Fig. 11 shows the PE for increasing 540 transmit power, within the region of power values used in 541 the communication standards for $(10 \times 8)$ and $(8 \times 4)$ MIMO 542 systems. It is assumed here that the noise variance is $\sigma^{2}=1$ to 543 indirectly account for the path loss (and, hence, the useful signal 544 power loss) experienced in real transmission. It can be seen that 545 the proposed DLT scheme outperforms SMX and RSM in terms 546 


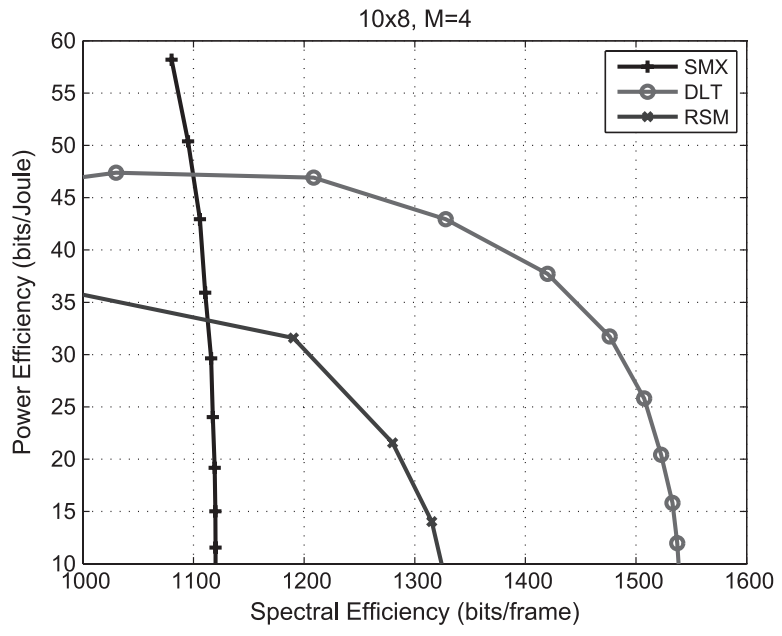

Fig. 12. Power efficiency versus spectral efficiency for a $(10 \times 8)$ MIMO with SMX and DLT, as well as QPSK with Rayleigh fading.

547 of PE for all transmit power values in both $(10 \times 8)$ and $(8 \times 4)$ 548 MIMO systems. The tradeoff between $\mathrm{PE}$ and $\mathrm{BE}$ is shown in 549 Fig. 12. It can be seen that DLT offers a more scalable tradeoff 550 with a wider range of $\mathrm{BEs}$ for the $\mathrm{PE}$ range, while it is more 551 power efficient than SMX and RSM in the region of high BEs.

\section{CONCLUSION}

553 A dual-layered DL transmission scheme was proposed, 554 which combines traditional MIMO SMX with RSM. As op555 posed to traditional SM where a subset of antennas carry a spa556 tial stream, here, we allow all antennas to carry information by 557 applying SM on the symbol power-level domain. This provides 558 scope for the analytical optimization of the ratio between the 559 power levels used in the proposed scheme. Both our simulations 560 and performance analysis show that, by allowing all antennas 561 to form spatial streams, the proposed scheme improves the 562 system's BE and power efficiency compared to both SMX 563 and SM.

564 Further work can involve exploring more advanced TPC 565 schemes for the proposed transmission scheme and exploring 566 the adaptations of the proposed scheme for QAM and enhanc567 ing its robustness to channel state information errors.

\section{REFERENCES}

11] D. Gesbert, M. Kountouris, R. Heath, C. Chae, and T. Salzer, "Shifting the MIMO paradigm," IEEE Signal Process. Mag., vol. 24, no. 5, pp. 36-46, Sep. 2007.

[2] M. Costa, "Writing on dirty paper,"IEEE Trans. Inf. Theory, vol. IT-29, no. 3, pp. 439-441, May 1983.

[3] C. Windpassinger, R. Fischer, T. Vencel, and J. Huber, "Precoding in multiantenna and multiuser communications," IEEE Trans. Wireless Commun., vol. 3, no. 4, pp. 1305-1316, Jul. 2004.

4] C. Masouros, M. Sellathurai, and T. Rantarajah, "Interference optimization for transmit power reduction in Tomlinson-Harashima precoded MIMO downlinks," IEEE Trans. Signal Process., vol. 60, no. 5, pp. 24702481, May 2012.

5] A. Garcia and C. Masouros, "Power loss reduction for MMSE-THP with multidimensional symbol scaling," IEEE Commun. Lett., vol. 18, no. 7, pp. 1147-1150, Jul. 2014.
[6] A. Garcia and C. Masouros, "Power-efficient Tomlinson-Harashima 584 precoding for the downlink of multi-user MISO systems," IEEE Trans. 585 Commun., vol. 62, no. 6, pp. 1884-1896, Jun. 2014.

[7] C. B. Peel, B. M. Hochwald, and A. L. Swindlehurst, "A vector- 587 perturbation technique for near-capacity multiantenna multiuser 588 communication-Part I: Channel inversion and regularization," IEEE 589 Trans. Commun., vol. 53, no. 1, pp. 195-202, Jan. 2005.

[8] C. Masouros and E. Alsusa, "Dynamic linear precoding for the exploita- 591 tion of known interference in MIMO broadcast systems," IEEE Trans. 592 Wireless Commun., vol 8, no. 3, pp. 1396-1404, Mar. 2009. 593

[9] C. Masouros and E. Alsusa, "Soft transmitter precoding for the down- 594 link of DS/CDMA communication systems," IEEE Trans. Veh. Technol., 595 vol. 59, no. 1, pp. 203-215, Jan. 2010.

[10] C. Masouros, T. Ratnarajah, M. Sellathurai, C. Papadias, and A. Shukla, 597 "Known interference in wireless communications: A limiting factor or a 598 potential source of green signal power?" IEEE Commun., Mag., vol. 51, 599 no. 10 , pp. $162-171$, Oct. 2013.

[11] G. Zheng et al., "Rethinking the role of interference in wireless networks," 601 IEEE Commun. Mag., vol. 52, no. 11, pp. 152,158, Nov. 2014.

[12] C. Masouros, "Correlation rotation linear precoding for MIMO broadcast 603 communications," IEEE Trans. Signal Process., vol 59, no. 1, pp. 252-262, 604 Jan. 2011.

[13] F. Rusek et al., "Scaling up MIMO: Opportunities and challenges with 606 very large arrays," IEEE Signal Process. Mag., vol. 30, no. 1, pp. 40-60, 607 Jan. 2013.

608

[14] C. Masouros, M. Sellathurai, and T. Ratnarajah, "Large-scale MIMO 609 transmitters in fixed physical spaces: The effect of transmit correlation and 610 mutual coupling," IEEE Trans. Commun., vol. 61, no. 7, pp. 2794-2804, 611 Jul. 2013.

612

[15] C. Masouros and M. Matthaiou, "Physically constrained massive MIMO: 613 Hitting the wall of favorable propagation," IEEE Commun. Let., vol. 19, 614 no. 5, pp. 771-774, May 2015.

[16] R. Mesleh, H. Haas, S. Sinanovic, C. W. Ahn, and S. Yun, "Spatial 616 modulation," IEEE Trans. Veh. Technol., vol. 57, no. 4, pp. 2228-2241, 617 Jul. 2008.

[17] M. Di Renzo, H. Haas, A. Ghrayeb, S. Sugiura, and L. Hanzo, "Spatial 619 modulation for generalized MIMO: Challenges, opportunities, and imple- 620 mentation," Proc. IEEE, vol. 102, no. 1, pp. 56-103, Jan. 2014.

621

[18] A. Garcia and C. Masouros, "Low-complexity compressive sensing detec- 622 tion for spatial modulation in large-scale multiple access channels," IEEE 623 Trans. Commun., to be published.

[19] M. Di Renzo and H. Haas, "Bit error probability of space modulation over 625 Nakagami-m fading: Asymptotic analysis," IEEE Commun. Lett., vol. 15, 626 no. 10 , pp. 1026-1028, Oct. 2011.

[20] J. Jeganathan, A. Ghrayeb, and L. Szczecinski, "Spatial modulation: 628 Optimal detection and performance analysis," IEEE Commun. Lett., 629 vol. 12, no. 8, pp. 545-547, Aug. 2008.

630

[21] A. Younis, S. Sinanovic, M. Di Renzo, R. Mesleh, and H. Haas, "Gen- 631 eralised sphere decoding for spatial modulation," IEEE Trans. Commun., 632 vol. 61, no. 7, pp. 2805-2815, Jul. 2013.

[22] J. Wang, S. Jia, and J. Song, "Generalised spatial modulation system 634 with multiple active transmit antennas and low complexity detection 635 scheme," IEEE Trans. Wireless Commun., vol. 11, no. 4, pp. 1605-1615, 636 Apr. 2012.

[23] M. Di Renzo and H. Haas, "On transmit diversity for spatial modulation 638 MIMO: Impact of spatial constellation diagram and shaping filters at the 639 transmitter," IEEE Trans. Veh. Technol., vol. 62, no. 6, pp. 2507-2531, 640 Jul. 2013.

641

[24] P. Yang et al., "Star-QAM signaling constellations for spatial mod- 642 ulation," IEEE Trans. Veh. Technol., vol. 63, no. 8, pp. 3741-3749, 643 Oct. 2014.

[25] S. Sugiura, C. Xu, S. X. Ng, and L. Hanzo, "Reduced-complexity coherent 645 versus non-coherent QAM-aided space-time shift keying," IEEE Trans. 646 Commun., vol. 59, no. 11, pp. 3090-3101, Nov. 2011.

[26] K. Ntontin, M. Di Renzo, A. Perez-Neira, and C. Verikoukis, "Adaptive 648 generalized space shift keying," EURASIP J. Wireless Commun. Netw., 649 vol. 2013, no. 1, p. 43, Feb. 2013.

[27] S. Sugiura and L. Hanzo, "On the joint optimization of dispersion 651 matrices and constellations for near-capacity irregular precoded space- 652 time shift keying," IEEE Trans. Wireless Commun., 2013, vol. 12, no. 1, 653 pp. 380-387, Jan. 2013.

[28] M. Maleki, H. Bahrami, S. Beygi, M. Kafashan, and N. H. Tran, "Space 655 modulation with CSI: Constellation design and performance evaluation," 656 IEEE Trans. Veh. Technol., vol. 62, no. 4, pp. 1623-1634, May 2013. 657

[29] X. Guan, Y. Cai, and W. Yang, "On the mutual information and precoding 658 for spatial modulation with finite alphabet," IEEE Wireless Commun. 659 Lett., vol. 2, no. 4, pp. 383-386, Aug. 2013. 
661 [30] J. M. Luna-Rivera, D. U. Campos-Delgado, and M. G. Gonzalez-Perez, 662 "Constellation design for spatial modulation," Procedia Technol., vol. 7, 663 pp. 71-78, 2013.

664 [31] C. Masouros, "Improving the diversity of spatial modulation in MISO 665 channels by phase alignment," IEEE Commun. Lett., vol. 18, no. 5, 666 pp. 729-732, May 2014.

667 [32] A. Garcia, C. Masouros, and L. Hanzo, "Pre-scaling optimization for space 668 shift keying based on semidefinite relaxation," IEEE Trans. Commun., 669 to be published.

670 [33] C. Masouros and L. Hanzo, "Constellation-randomization achieves 671 transmit diversity for single-RF spatial modulation," IEEE Trans. Veh. 672 Technol., to be published.

673 [34] J. Jeganathan, A. Ghrayeb, L. Szczecinski, and A. Ceron, "Space shift 674 keying modulation for MIMO channels," IEEE Trans. Wireless Commun., 675 vol. 8, no. 7, pp. 3692-3703, Jul. 2009.

676 [35] M. Di Renzo and H. Haas, "Bit error probability of space modulation over 677 Nakagami-m fading: Asymptotic analysis," IEEE Commun. Lett., vol. 15, 678 no. 10, pp. 1026-1028, Oct. 2011.

679 [36] R. Zhang, L. Yang, and L. Hanzo, "Generalised pre-coding aided spatial 680 modulation," IEEE Trans. Wireless Commun., vol. 12, no. 11, pp. 54346815443 , Nov. 2013.

682 [37] D.-T. Phan-Huy and M. Helard, "Receive antenna shift keying for 683 time reversal wireless communications," in Proc. IEEE ICC, Jun. 2012, 684 pp. $4852-4856$.

685 [38] R. Zhang, L. L. Yang, and L. Hanzo, "Error probability and capacity 686 analysis of generalised pre-coding aided spatial modulation," IEEE Trans. 687 Wireless Commun., vol. 14, no. 1, pp. 364-375, Jan. 2015

688 [39] A. Stavridis, S. Sinanovic, M. Di Renzo, and H. Haas, "Transmit precod689 ing for receive spatial modulation using imperfect channel knowledge," in 690 Proc. IEEE Veh. Technol. Conf. Spring, May 2012, pp. 1-5.

691 [40] R. M. Legnain, R. H. M. Hafez, I. D. Marsland, and A. M. Legnain, 692 "A novel spatial modulation using MIMO spatial multiplexing," in Proc. 693 ICCSPA, Feb. 2013, pp. 1-4.

694 [41] J. G. Proakis, Digital Communications, Electrical Engineering, 3rd ed. 695 New York, NY, USA: McGraw-Hill, 1995.

696 [42] M. Abramowitz and I. A. Stegun, Handbook of Mathematical Functions 697 With Formulas, Graphs, and Mathematical Tables. New York, NY, USA: 698 Dover, 1972.

699 [43] X. Cong, G. Y. Li, Z. Shunqing, Y. Chen, and S. Xu, "Energy- and 700 spectral-efficiency tradeoff in downlink OFDMA networks," IEEE Trans. 701 Wireless Commun., vol. 10, no. 11, pp. 3874-3886, Nov. 2011.

702 [44] S. Cui, A. J. Goldsmith, and A. Bahai, "Energy-constrained modulation 703 optimization," IEEE Trans. Wireless Commun., vol. 4, no. 5, pp. 23497042360, Sep. 2005.

705 [45] C. Masouros, M. Sellathurai, and T. Ratnarajah, "Computationally ef706 ficient vector perturbation precoding using thresholded optimization," 707 IEEE Trans. Commun., vol. 61, no. 5, pp. 1880-1890, May 2013.

708 [46] C. Masouros, M. Sellathurai, and T. Ratnarajah, "Vector perturbation 709 based on symbol scaling for limited feedback MIMO downlinks," IEEE 710 Trans. Signal Process., vol. 62, no. 3, pp. 562-571, Feb. 1, 2014.

711 [47] D. Curd, Power consumption in 65 nm FPGAs, Xilinx White Paper, 712 Feb. 2007.

713 [48] Evolved Universal Terrestrial Radio Access (E-UTRA); LTE Physical 714 Layer; General Description, Third-Generation Partnership Project, TS $715 \quad 36.201$, V11.1.0 (2008-03), Rel. 11, 2008.

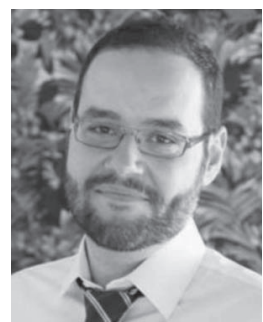

Christos Masouros (M'06-SM'14) received the 716 Diploma in electrical and computer engineering 717 from the University of Patras, Patras, Greece, in 718 2004 and the M.Sc. and Ph.D. degrees in electrical 719 and electronic engineering from The University of 720 Manchester, Manchester, U.K., in 2006 and 2009, 721 respectively.

722

He was a Research Associate with the University 723 of Manchester and a Research Fellow with Queen's 724 University Belfast, Belfast, U.K. He is currently a 725 Lecturer with the Department of Electrical and Elec- 726 tronic Engineering, University College London, London, U.K. His research in- 727 terests include wireless communications and signal processing, with particular 728 focus on green communications, large-scale antenna systems, cognitive radio, 729 and interference mitigation techniques for multiple-input-multiple-output and 730 multicarrier communications.

Dr. Masouros is the Principal Investigator of the EPSRC Project 732 EP/M014150/1 on large-scale antenna systems. He received a Royal Academy 733 of Engineering Research Fellowship for 2011-2016.

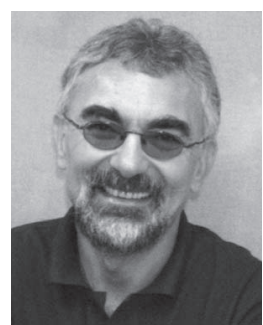

Lajos Hanzo (M'91-SM'92-F'04) received the 735 M.S. degree in electronics and the Ph.D. degree from 736 Budapest University of Technology and Econom- 737 ics (formerly, Technical University of Budapest), 738 Budapest, Hungary, in 1976 and 1983, respectively; 739 the D.Sc. degree from the University of Southampton, 740 Southampton, U.K., in 2004; and the "Doctor Honoris 741 Causa" degree from Budapest University of Technol- 742 ogy and Economics in 2009.

743

During his 38-year career in telecommunications, 744 he has held various research and academic posts in 745 Hungary, Germany, and the U.K. Since 1986, he has been with the School 746 of Electronics and Computer Science, University of Southampton, where he 747 holds the Chair in Telecommunications. He is currently directing a 100-strong 748 academic research team, working on a range of research projects in the field of 749 wireless multimedia communications sponsored by industry, the Engineering 750 and Physical Sciences Research Council of U.K., the European Research 751 Council's Advanced Fellow Grant, and the Royal Society Wolfson Research 752 Merit Award. During 2008-2012, he was a Chaired Professor with Tsinghua 753 University, Beijing, China. He is an enthusiastic supporter of industrial and 754 academic liaison and offers a range of industrial courses. He has successfully 755 supervised more than $80 \mathrm{Ph} . D$. students, coauthored 20 John Wiley/IEEE Press 756 books on mobile radio communications, totaling in excess of 10000 pages, and 757 published more than 1400 research entries on IEEEXplore. He has more than 758 20000 citations. His research is funded by the European Research Council's 759 Senior Research Fellow Grant.

Dr. Hanzo is a Fellow of the Royal Academy of Engineering, The Institution 761 of Engineering and Technology, and the European Association for Signal 762 Processing. He is also a Governor of the IEEE Vehicular Technology Society. 763 He has served as the Technical Program Committee Chair and the General Chair 764 of IEEE conferences, has presented keynote lectures, and has been awarded a 765 number of distinctions. During 2008-2012, he was the Editor-in-Chief of the 766 IEEE Press. 


\section{AUTHOR QUERIES}

\section{AUTHOR PLEASE ANSWER ALL QUERIES}

AQ1 = Please provide publication update in Ref. [18].

AQ2 = Please provide publication update in Ref. [32].

AQ3 = Please provide publication update in Ref. [33].

\section{END OF ALL QUERIES}

